\title{
Le contrôle des projets d'innovation de haute technologie
}

\author{
Nicolas BERLAND \\ Professeur \\ Université Paris-Dauphine, DRM-Crefige \\ Place du Maréchal de Lattre de Tassigny \\ 75775 Paris Cedex 16 \\ nicolas.berland@free.fr \\ François PERSIAUX \\ Docteur en gestion, HEC \\ 17, impasse du Bua \\ 92160 Antony \\ fpersiaux@orange.fr
}

\section{Résumé :}

Le contrôle de gestion d'une innovation est une problématique délicate, et pour certains insoluble, en particulier lorsque le développement de nouveaux produits ou services s'appuie sur des technologies dont le modèle économique est peu connu. Toutefois, l'article présente un cadre d'analyse, fondé sur les analyses de Simons (1995), pour comprendre comment des chefs de projet impliqués dans la gestion d'un projet d'innovation de haute technologie s'y prennent pour gérer une innovation. Ce cadre d'analyse est ensuite mobilisé sur quatre études de cas longitudinales. L'article montre ainsi que des styles de gestion et des modèles existent que fait ressortir notre grille d'analyse.

\section{Mots-clés :}

Contrôle, innovation, gestion de projet, pilotage, contrôle interactif et diagnostique

\section{Management control of technological innovation projects}

\begin{abstract}
:
Management control is a very difficult task when applied to innovation. For some researchers, it's even an impossible mission. Despite this, our paper presents a theoretical framework issued from a literature review and based on Simons research (1995). This framework is used to understand how project managers involved in the management of technological innovation projects succeed to manage. The paper shows how on a very broad perspective, we can't distinguish project managers' behaviours but with a more detailed analysis several patterns can be identified.
\end{abstract}

\section{Keywords:}

Control, innovation, project management, interactive and diagnostic control

Adresse de correspondance : Nicolas Berland, Université Paris-Dauphine, DRM-Crefige, Place du Maréchal de Lattre de Tassigny, 75775 Paris Cedex 16 


\section{Le contrôle des projets d'innovation de haute technologie}

\section{INTRODUCTION}

Contrôle de gestion et innovation font-ils bon ménage ? Leur coexistence paraît aussi délicate que celle des fonctions de contrôle de gestion et de Recherche et Développement. Si le divorce n'a jamais été prononcé, c'est peut-être tout simplement parce que le mariage n'a jamais eu lieu (Abernathy et Brownell, 1999). La rigueur et la créativité, si caractéristiques a priori de l'une et de l'autre des deux fonctions, ne peuvent cohabiter que très difficilement. Alter (1998) propose ainsi de voir l'innovation et l'organisation comme deux légitimités contradictoires. Latour (1987), Akrich, Callon et Latour (1988) décrivent quant à eux l'illusion du contrôle d'un processus de recherche et d'innovation qui obéit à ses propres logiques et reste largement indéterminé.

Pourtant, certains chercheurs ne s'intéressent qu'à ce thème (Van de Ven \& al., 1989, Vinck \& al., 1991, Zaltman \& al., 1973). L'une des difficultés est que le concept de contrôle pertinent pour ce type d'activité n'est vraisemblablement pas un contrôle de type «cybernétique » tel que défini par Hofstede (1981) car les buts à atteindre sont trop incertains. La distinction introduite par Simons (1995a et b) entre contrôle interactif et contrôle diagnostique est en revanche porteuse de nouvelles ouvertures théoriques propres à enrichir la réflexion sur ce domaine. De même, il semble que, dans de nombreuses études, le contrôle de gestion ait essentiellement été appréhendé sous l'angle de la comptabilité de gestion alors que des définitions plus organisationnelles et moins comptables peuvent être retenues. La définition du contrôle à laquelle nous faisons appel dans le cadre de l'étude des projets d'innovation étudiés est donc celle, relativement large, proposée par Chiapello (1996), "le contrôle est toute influence créatrice d'ordre, c'est-à-dire 
d'une certaine régularité », sans plus de précisions quant au caractère exhaustif d'une telle influence. Cette définition nous paraît fructueuse pour traiter notre question de recherche: comment un chef de projet peut-il contrôler un projet d'innovation de haute technologie ?

Pour répondre à cette question, nous avons étudié, pendant quatre ans, dans le cadre d'études de cas, quatre Projets d'Innovation de Haute Technologie (PIHT) dans une même entreprise. Pour chacun de ces projets, nous avons analysé les pratiques de contrôle mises en place par les chefs de projet pour des innovations de haute technologie.

Nous inscrivons notre recherche dans la continuité de l'approche développée par ECOSIP ${ }^{1}$ (Giard et Midler, 1993). Cet ouvrage qui montre la diversité des recherches et illustrait l'importance de la gestion par projet dans ce domaine a été enrichi par un deuxième ouvrage (Cohendet, Jacot et Lorino, 1996) mettant la gestion de projet d'innovation en perspective puis par un troisième (Garel, Giard et Midler, 2004) présentant les renouvellements récents du management de projet. Nous croiserons ces approches avec le cadre théorique de Simons suivant en cela des travaux récents notamment développés par Davila (2000) ou Gautier (2002). Il s'agit alors d'élargir le champ du contrôle et de comprendre comment les chefs de projet peuvent l'utiliser autrement que comme un outil cybernétique fondé sur des adaptations en simple boucle par rapport à des buts prédéfinis. Mais alors que Tani (1995) et Davila (2000) se sont beaucoup intéressés aux variables de contingence qui influençaient l'incertitude et l'interactivité, nous nous situerons plutôt dans la veine des travaux de Nixon (1998) et Gautier (2002) qui cherchent à comprendre comment fonctionne l'interactivité, sans pour autant rejeter les approches contingentes précédentes. Dans ces deux études, l'interactivité permet d'accroître la qualité de la communication et permet au projet de mieux fonctionner. Nixon (1998) montre par exemple comment l'interactivité change au 
cours du temps faisant que les managers utilisent d'abord des critères qualitatifs de décision puis des critères quantitatifs.

A l'issue de cette introduction, la seconde partie définit les bornes de notre objet d'étude et les problématiques de recherche qui y sont associées. La troisième partie présentera le cadre d'analyse retenu pour observer les pratiques en usage dans nos cas. La quatrième partie sera consacrée à la présentation de la méthodologie et de nos études de cas. La cinquième partie présentera les résultats obtenus.

\section{GESTION DE L'INNOVATION, GESTION DE PROJET : AU-DELA DU CONTROLE CYBERNETIQUE}

Il est nécessaire de clairement identifier les innovations qui sont l'objet de cette recherche et les modes de gestion qui les caractérisent afin de délimiter notre objet d'étude: les projets d'innovation de haute technologie (PIHT). Nous décrirons ensuite comment ces objets peuvent être étudiés dans le cadre théorique défini par Simons.

\subsection{LE CHOIX DE L'INNOVATION DE HAUTE TECHNOLOGIE}

Nous nous intéressons dans cet article à des activités d'innovation de haute technologie. L'innovation peut être définie au sens de Schumpeter (1955) «comme la première transaction commerciale réussie, par opposition à l'invention ». C'est le couplage, réalisé par les acteurs, entre une technique et un marché qui est notre objet d'étude. Celui-ci se définit par plusieurs caractéristiques :

\footnotetext{
${ }^{1}$ Economie et Systèmes Intégrés de Production, un réseau de recherche français.
} 
- la «haute » technologie signifie que la nouveauté des techniques mises en œuvre ou la complexité de leur assemblage caractérise ces activités. Cela différencie notre objet de recherche des innovations uniquement fondées sur des dimensions marketing ou organisationnelles. Nous sommes alors assez proches des innovations observables dans les secteurs pharmaceutiques, aéronautiques ou automobiles. Les principales fonctions de l'entreprise impliquées sont dès lors la Recherche et Développement, l'Ingénierie et le Marketing.

- En reprenant la distinction utilisée par Lenfle (2004), notre objet de recherche n'est plus une activité de recherche. Ce n'est pas non plus une activité de développement, dans le sens où les protagonistes ne savent pas encore ce qu'ils veulent précisément développer.

L'activité d'innovation de haute technologie pose des problèmes spécifiques de contrôle de gestion : "l'innovation ne peut pas être programmée (...) car les modalités de l'action ne se découvrent qu'en marchant : (...) c'est en avançant dans un projet que l'on découvre les supports humains qui s'y associent et le rendent finalement effectif »(Alter, 1995). Cette limite est atteinte autant pour les innovations dites « radicales»-Zaltman \& al. (1973) les définissent comme une « réorientation des recherches passées ou en cours»-que pour les innovations dites «progressives »- définie par les mêmes auteurs comme «une variation des recherches passées ou en cours ». Nous ne sommes donc pas en mesure de limiter a priori l'étude à l'un ou l'autre de ces types d'innovation.

Au-delà, plusieurs raisons justifient notre choix pour un tel objet de recherche : 
1. Il n'y a pas de répétitivité de l'activité d'innovation (Giard et Midler, 1993). Toute modélisation est donc incertaine, en particulier le «temps de mise sur le marché », alors même qu'il s'agit le plus souvent d'un enjeu stratégique majeur.

2. Il est difficile pour les chercheurs, les ingénieurs, les hommes de marketing ou les comptables de gestion de disposer d'une référence technico-économique réutilisable.

3. La durée de gestation d'une innovation est généralement courte - moins de cinq ans - et n'a a priori que peu de liens avec les cycles budgétaires annuels et les clôtures comptables.

4. Enfin, le caractère transverse aux différentes fonctions ou départements d'entreprise renforcé notamment par un mode de conception en ingénierie «simultanée »- pose la question des responsabilités diluées et partagées dans l'entreprise, ce qui est a priori antinomique avec les notions de délégation et de centre de responsabilité typiques du contrôle de gestion traditionnel (Bouquin, 1994).

Les activités d'Innovation de Haute Technologie que nous étudions sont en outre gérées en mode projet.

\subsection{LA GESTION PAR PROJET DES INNOVATIONS DE HAUTE TECHNOLOGIE}

Quels sont les mécanismes permettant de contrôler le développement d'une innovation? Galbraith (1977) propose une synthèse des différents moyens possibles d'intégration des fonctions : contacts directs, fonctions de liaison, groupes de travail, équipes projets, fonction de médiateur, fonction de direction transverse et enfin organisation matricielle. Cette analyse n'implique pas nécessairement que la gestion par projet soit un mode d'organisation des activités d'innovation de haute technologie plus favorable à l'innovation que les groupes de travail ou la 
fonction de médiateur. C'est pourtant le choix de gestion principal qui a été fait par l'entreprise que nous étudions et un choix relativement courant dans ce type d'activités, comme l'indique notamment des auteurs comme Clark \& Wheelwright (1992). Quelles en sont alors les dimensions saillantes en termes de contrôle?

Le management de projet est un mode de gestion largement étudié (Garel, 2003) et nous nous limiterons ici volontairement à l'intersection de ce champ disciplinaire avec celui du contrôle. Un projet est défini par Anthony (1988) comme «une activité non récurrente créée pour atteindre un objectif spécifique ». Anthony, Dearden et Govindarajan (1992) précisent cette définition : «un projet est un ensemble d'activités dont l'objectif est d'accomplir un résultat final défini de suffisamment d'importance pour intéresser l'encadrement. »Dans un domaine où l'incertitude technique et commerciale est relativement forte, un projet d'innovation de haute technologie (PIHT) peut alors être défini comme une entité non pérenne d'une entreprise dotée d'une autorité formalisée, dont l'objet est de concentrer les énergies pour concevoir un produit ou un service innovant, différencié par les clients de l'entreprise grâce à sa technologie.

De nombreuses manières de caractériser les PIHT sont proposées par la littérature de gestion de projet et par la littérature spécialisée dans l'innovation technologique :

- $\quad$ taille du projet et structure juridique (Giard et Midler, 1993), avec notamment la typologie A (projet «majeur» au sein d'une entreprise), B (projet inter-entreprises, dans le cadre d'alliances en particulier) et C (projet « ordinaire »d'une entreprise),

- projets indépendants les uns des autres ou non (Danila, 1985), 
- ou encore projets de type «poussé par la technologie» ou «tiré par le marché » (Courpasson et Gaillard, 1991).

Ces caractérisations sont autant de facteurs de contingence que nous avons intégrés à notre analyse des pratiques de contrôle possibles des PIHT.

L'innovation technologique et le management de projet permettent globalement de remettre en question la conception traditionnelle du «contrôle» tant il s'agit d'activités incertaines, peu répétitives et organisées par essence de manière collective et transverse.

\subsection{LE CONTROLE INTERACTIF COMME CADRE D’ANALYSE DES PIHT}

Simons $(1990 ; 1991 ; 1994)$ constate que «toutes les organisations grandes et complexes ont des systèmes similaires de contrôle de gestion [...] mais qu'il y a des différences dans la manière de les utiliser » (Simons, 1990, p.135). Il en vient à caractériser les différences entre les systèmes de contrôle par la façon dont les managers utilisent activement certains outils de contrôle alors qu'ils délèguent les autres. Il différencie ainsi, dans une série d'écrits (Simons, 1987, 1990, 1991, 1994, 1995a, b), ce qu'il appelle le contrôle interactif du contrôle diagnostique. Dans son ouvrage de synthèse, Simons (1995a, 1995b) définit les systèmes de «contrôle diagnostique » comme « les systèmes d'information formels que les managers utilisent pour surveiller les résultats de l'organisation et corriger les déviations par rapport aux standards prédéfinis de performance » (Simons, 1995b, p.59) et les systèmes de «contrôle interactif » comme «les systèmes formels d'information que les managers utilisent pour s'impliquer régulièrement et personnellement dans les décisions de leurs subordonnés »(Simons, 1995b, p.95). Cette vision des systèmes de contrôle a aujourd'hui un retentissement certain (Abernethy et Brownell, 1999 ; Bisbe et Otley, 2004 ; Bruining et al., 2004 ; Marginson, 2002 ; Osborn, 1998). Selon Simons, «la quasi-totalité des 
écrits en contrôle de gestion se réfèrent à des systèmes de contrôle diagnostique » (1995b, p.60). Le contrôle diagnostique s'apparenterait donc à ce qu'on appelle classiquement «contrôle de gestion » (Simons, 1995b, p.61). Ce type de système permet à l'entreprise de fonctionner sans surveillance constante car les managers ne s'y impliquent que s'il y a des écarts par rapport aux résultats attendus : c'est le principe du management par exception.

C'est ce contrôle cybernétique qui nous paraît inadapté au management de projet et à l'innovation. C'est donc au contrôle interactif qu'il semble préférable de s'intéresser pour renouveler les réflexions sur la discipline. Or, cette démarche a précisément rencontré un certain succès appliquée aux projets ces dernières années (Tani, 1995, Nixon, 1998, Davila, 2000, Gautier, 2002).

Simons considère qu'il ne peut y avoir trop de systèmes de contrôle utilisés simultanément de manière interactive. En effet, ce type de contrôle a un coût économique et cognitif (les individus ne peuvent prêter attention à tout). Se concentrer sur trop de systèmes de contrôle en même temps risque de conduire à une surcharge d'informations, des analyses superficielles et une paralysie. Simons constate que les dirigeants sélectionnent les modes de contrôle interactifs à mettre en place en fonction de la stratégie et des incertitudes stratégiques à maîtriser (Simons, 1990, 1991). Ces choix ont, au final, une incidence sur l'information diffusée, l'apprentissage organisationnel et les stratégies émergentes.

Mais les mécanismes par lesquels les outils de contrôle interactifs ont une incidence sur la stratégie restent peu documentés (Marginson, 2002). Ainsi, Simons ne donne que très peu d'éléments sur la manière dont ces outils de contrôle interactifs sont utilisés dans les organisations. Il considère que le simple fait que la direction focalise son attention sur un outil permet de le rendre interactif. Les modalités de fonctionnement d'un système de contrôle de 
gestion interactif et diagnostique restent donc à explorer. Comme le montre d'ailleurs Gautier (2002), l'interactivité verticale (entre dirigeants et subordonnés) se double en gestion de projet d'une interactivité horizontale (entre les membres de l'équipe projet). Le fonctionnement d'un outil de contrôle interactif mérite ainsi un examen plus approfondi. Notre question n'est donc pas de savoir pourquoi les entreprises adoptent un schéma particulier de contrôle mais plutôt comment et selon quelles modalités elles le font. Selon Gautier et Lenfle (2004), la fréquence et l'intensité des communications peuvent expliquer le succès et l'échec d'un projet de conception et de développement d'un produit nouveau. C'est précisément le point que nous cherchons à étudier ici en nous appuyant sur l'observation des comportements managériaux en matière de contrôle.

Afin d'étudier les modalités de contrôle interactif, nous avons construit, à partir de la littérature préexistante, un outil d'observation des pratiques managériales de contrôle applicable à notre objet d'étude.

\section{LES MOYENS DE CONTROLE POSSIBLES D'UN PROJET D'INNOVATION DE HAUTE TECHNOLOGIE}

Ayant défini notre objet d'étude et le cadre théorique de référence, nous devons maintenant identifier les modalités possibles de contrôle d'un projet d'innovation. Trop souvent, le contrôle des projets d'innovation est étudié dans une perspective exclusive de comptabilité de gestion. Or nous souhaitons l'élargir au contrôle organisationnel. Dans le cadre de cet article, nous avons ordonné les pratiques de contrôle dans un cadre d'analyse permettant de mesurer comment les acteurs influencent les activités d'innovation de haute technologie. La grille d'analyse que nous allons construire constitue le premier apport théorique de notre article. 


\subsection{LES AXES PRINCIPAUX DU CADRE D'ANALYSE DES MOYENS DE CONTROLE DE PIHT}

Partant du constat que «de nombreuses typologies listant les différents types de contrôle possibles dans les entreprises ont été produites dans l'histoire de la théorie des organisations », Chiapello (1996) "propose un modèle d'analyse permettant de réconcilier les différents travaux». Une fois le contrôle défini comme "toute influence créatrice d'ordre, c'est-à-dire d'une certaine régularité », ce modèle propose une grille d'analyse des modes de contrôle définis comme «facteurs d'influence particuliers » ou «modalités d'exercice du contrôle ». C'est dans ce cadre que nous souhaitons définir notre propre méthode de classification des Moyens de Contrôle de PIHT proposés par la littérature spécialisée. Le modèle d'analyse de Chiapello s'appuie sur les six axes de classification suivants :

(6) Quels sont les moyens du contrôle?

(1) Qui contrôle ?

-le marché

-l'organisation

-règlements

-contrôle de gestion

-structure

-l'organisation

- machine

-administration

-la culture

-de l'organisation

-de la société

-des professionnels

-les relations inter-individuelles

une personne

-structure

-un groupe de personnes

-soi-même

(5)Quels sont les processus de contrôle

-cybernétiques, homéostatiques

-non cybernétiques :

-modèle politique

-modèle de la poubelle

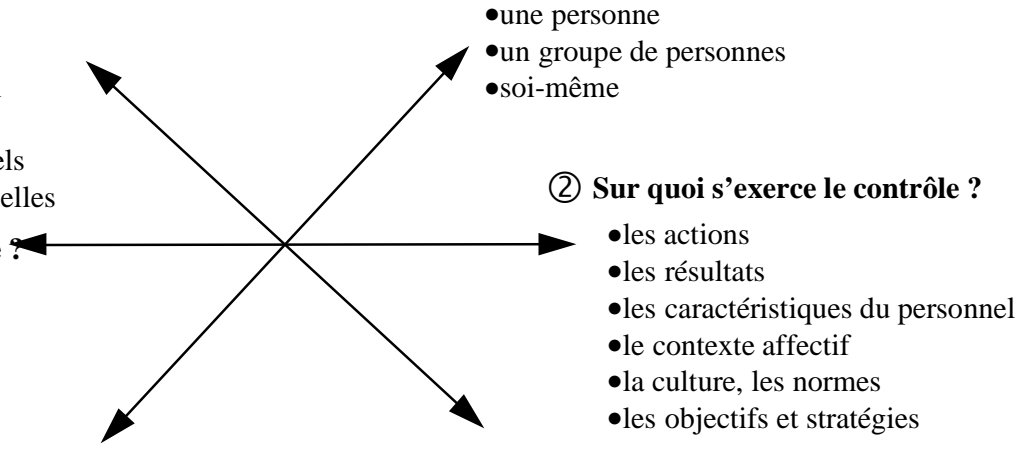

(4)Quand le contrôle a-t-il eu lieu ?

(3) Quelle est l'attitude du contrôlé ?

-avant l'action

- pendant l'action

-après l'action

-implication morale

- réalisation instrumentale

-aliénation 
Figure 1 : les caractéristiques de chacun des six axes de classification des modes de contrôle,

\section{Chiapello (1996)}

Dans le type de recherche que nous menons, les axes 1 , 3 , et 5 ne nous ont pas permis de différencier de manière significative les modes de contrôle des différents projets entre eux. Nous nous appuyons donc principalement sur l'axe 2 («sur quoi s'exerce le contrôle? ?) et sur l'axe 6 («quels sont les moyens de contrôler?») pour décrire les modes de contrôle de PIHT car ces deux dimensions se retrouvent dans tous projets. Nous posons ces questions à l'aide du fil conducteur que constitue l'axe temporel (axe 4: «quand le contrôle a-t-il eu lieu ?»), car les projets que nous observons s'étalent dans le temps.

\subsection{Les deuX grandes Strategies de controle de PIHT Proposees par la}

\section{LITTERATURE}

Pour définir l'ensemble des Moyens de Contrôle possibles de PIHT (axe 6 de la figure 1), nous avons passé en revue une vaste littérature traitant du contrôle de l'innovation et des projets. Nous avons ainsi repéré dans différents articles de recherche les Moyens de Contrôle auxquels un chef de projet peut recourir. Nous avons principalement utilisé les publications listées dans le tableau 1 ci-dessous dans lesquelles nous avons identifié des articles décrivant des MC. Nous indiquons dans ce tableau le nombre d'articles faisant référence explicitement à des $\mathrm{MC}$ possibles ${ }^{2}$ :

\begin{tabular}{|l|c|}
\hline \multicolumn{1}{|c|}{ Titre de la publication } & $\begin{array}{c}\text { Nombre } \\
\text { d'articles } \\
\text { utilisés }\end{array}$ \\
\hline R\&D Management & 41 \\
\hline Revue Française de Gestion & 18 \\
\hline Journal of Product Innovation Management & 12 \\
\hline
\end{tabular}

\footnotetext{
${ }^{2}$ Pour des questions de taille de l'article, nous ne donnons pas les références précises de tous les articles utilisés. Le lecteur pourra les trouver dans Persiaux (2005).
} 


\begin{tabular}{|l|c|}
\hline Research Technology Management & 12 \\
\hline Accounting, Organisation \& Society & 12 \\
\hline Revue Française de Comptabilité & 10 \\
\hline Gérer et Comprendre & 9 \\
\hline Gestion 2000 & 6 \\
\hline IEEE Transactions on Engineering Management & 6 \\
\hline
\end{tabular}

Tableau 1 : dénombrement des références par publication pour la définition des Moyens de Contrôle possibles

Cependant, dans un effort de classification, de nombreux MC portent sur un même thème, par exemple «Confier la maîtrise d'ouvrage projet à des clients internes de l'innovation » et «S'assurer du soutien d'un champion de l'innovation ayant du pouvoir dans l'organisation ». De tels Moyens de Contrôle sont donc regroupés dans une «famille», dans cet exemple « Rechercher l'intégration des fonctions de l'entreprise ».

Plus généralement, ces familles de Moyens de Contrôle ne portent pas systématiquement sur les mêmes objets de contrôle (axe 2 de la figure 1). Deux stratégies de contrôle distinctes sont en effet généralement distinguées : «piloter les moyens en cours d'exécution » et «maîtriser le résultat du projet». En première approche, il apparaît que «le pilotage des moyens en cours d'exécution» d'un PIHT renvoie directement à la notion de «processus » comme première stratégie du contrôle de PIHT. Ce terme "processus» peut être compris ici comme un enchaînement relativement ordonné de faits et d'actions, répondant à un certain schéma et aboutissant à un résultat en partie déterminé. La gestion de projet cherche en effet par construction à ordonner les actions des différentes fonctions de l'entreprise ( $R \& D$, ingénierie, marketing, contrôle de gestion...) au fur et à mesure que l'innovation se définit. Il s'agit de focaliser l'attention sur la mise en œuvre des moyens nécessaires à l'innovation et donc sur le processus d'innovation de haute technologie. 
Mais la gestion de projet cherche tout autant à «maitriser le résultat du projet », par conséquent à s'assurer du « résultat » de PIHT. Le résultat repose sur une mesure des productions de l'activité, un compte de résultat de l'innovation commercialisée conçue par le projet par exemple. Des auteurs plus spécialisés dans l'innovation de haute technologie comme Meyers et Wilemon (1989) font directement écho à cette notion en expliquant que le résultat atteint par un projet est difficile à préciser a priori mais que l'objectif d'innovation se précise généralement au fur et à mesure que les acteurs de l'innovation réagissent à l'évolution des prototypes successifs. Le « résultat » est donc la seconde stratégie importante du contrôle de PIHT.

Enfin, chacune de ces stratégies s'applique à des phases d'avant-projet, de réalisation et d'évaluation qui correspondent aux moments principaux du cycle de vie d'un projet (axe 4 de la figure 1). Les recherches en gestion de projet (Gautier et Lenfle, 2004, Gautier, 2004) montrent que les projets se concentrent sur trois phases de gestion principales partiellement concourantes : l'avant-projet ou fuzzy front end, la réalisation et l'évaluation. De fait, les deux stratégies de contrôle sont mises en œuvre durant les phases d'avant-projet, de réalisation et d'évaluation qui correspondent aux moments principaux du cycle de vie d'un projet. La phase d'avant-projet est l'une des moins étudiée par la littérature (Gautier et Lenfle, 2004) mais c'est dans cette phase que vont être établis les critères et les priorités qui permettent ensuite des arbitrages dans la phase de réalisation ou conception (Gautier et Lenfle, 2004). L'avant-projet se termine par une décision stratégique d'entreprendre le projet ou non. C'est l'une des particularités de notre recherche d'analyser le contrôle dans toute la durée du projet. 
En conclusion, avant d'aboutir à une classification exhaustive des Moyens de Contrôle possibles de PIHT, nous pouvons représenter l'ébauche de notre cadre d'analyse des Moyens de Contrôle possibles :

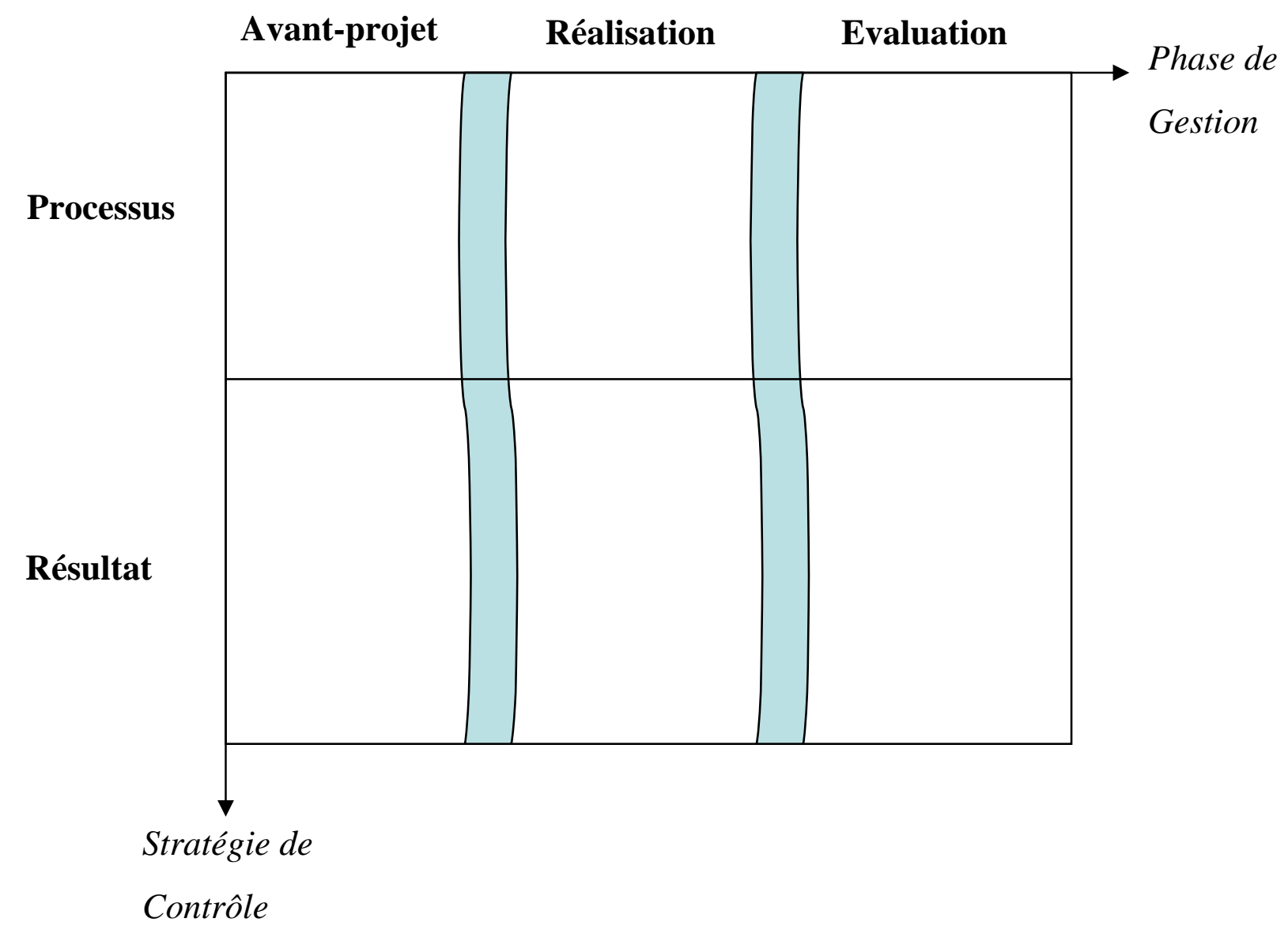

Figure 2 : cadre d'analyse des moyens possibles de contrôle de PIHT

Notre recherche permet de proposer une grille de collecte systématique des Moyens de contrôle de PIHT. Les deux principales stratégies du contrôle de PIHT repérées à ce stade sont le processus et le résultat de PIHT. 


\subsection{L'ARbTIRAge, UNE STRATEgIE INTERMEdiAire ENTRE LE PROCESSUS ET LE}

\section{RESUltat DE PIHT}

A ce stade, la stratégie de contrôle du «processus » de PIHT se décline en neuf familles de Moyens de Contrôle - les familles 1 à 9 du tableau ci-dessous - et la stratégie de contrôle du « résultat » d'un PIHT en dix familles de Moyens de Contrôle - les familles 4 à 13 du tableau cidessous.

\begin{tabular}{|c|c|c|c|c|c|c|}
\hline & $\begin{array}{l}\text { Associé au } \\
\text { processus }\end{array}$ & $\begin{array}{l}\text { Associé au } \\
\text { résultat }\end{array}$ & Famille de Moyens de Contrôle & Moyens & Objectifs & Résultats \\
\hline 1 & $\mathrm{X}$ & & $\begin{array}{l}\text { Favoriser la culture innovatrice des acteurs de } \\
\text { l'entreprise }\end{array}$ & \multicolumn{2}{|c|}{$\Leftrightarrow$} & \\
\hline 2 & $\mathrm{X}$ & & $\begin{array}{l}\text { Contenir la différenciation des fonctions de } \\
\text { l'entreprise }\end{array}$ & \multicolumn{2}{|c|}{$\Leftrightarrow$} & \\
\hline 3 & $\mathrm{X}$ & & $\begin{array}{l}\text { Rechercher l'intégration des fonctions de } \\
\text { l'entreprise }\end{array}$ & \multicolumn{2}{|c|}{$\Leftrightarrow$} & \\
\hline 4 & $\mathrm{X}$ & $\mathrm{X}$ & Gérer le slack & \multicolumn{3}{|c|}{$\Leftrightarrow$} \\
\hline 5 & $\mathrm{X}$ & $\mathrm{X}$ & $\begin{array}{l}\text { Piloter la qualité au sein de l'entreprise en } \\
\text { concevant de façon pragmatique }\end{array}$ & \multicolumn{3}{|c|}{$\Leftrightarrow$} \\
\hline 6 & $\mathrm{X}$ & $\mathrm{X}$ & Evaluer le projet & \multicolumn{3}{|c|}{$\Leftrightarrow$} \\
\hline 7 & $\mathrm{X}$ & $\mathrm{X}$ & Piloter l'allocation des ressources aux tâches & \multicolumn{3}{|c|}{$\Leftrightarrow$} \\
\hline 8 & $\mathrm{X}$ & $\mathrm{X}$ & Piloter les coûts de projet & \multicolumn{3}{|c|}{$\Leftrightarrow$} \\
\hline 9 & $\mathrm{X}$ & $\mathrm{X}$ & Arbitrer entre des dimensions coûts/délais/qualités & \multicolumn{3}{|c|}{$\Leftrightarrow$} \\
\hline 10 & & $\mathrm{X}$ & Piloter les délais du projet & \multicolumn{3}{|c|}{$\Leftrightarrow$} \\
\hline 11 & & $\mathrm{X}$ & $\begin{array}{l}\text { Piloter la qualité perçue du produit ou service par } \\
\text { un client externe à l'entreprise }\end{array}$ & \multicolumn{3}{|c|}{$\Leftrightarrow$} \\
\hline 12 & & $\mathrm{X}$ & $\begin{array}{l}\text { Piloter le chiffre d'affaires prévisionnel du produit } \\
\text { ou service développé }\end{array}$ & \multicolumn{3}{|c|}{$\Leftrightarrow$} \\
\hline 13 & & $\mathrm{X}$ & Piloter les coûts de l'objet développé & \multicolumn{3}{|c|}{$\Leftrightarrow$} \\
\hline
\end{tabular}

Tableau 2 : associations possibles entre les stratégies de contrôle et les familles de MC repérées jusqu'ici

Les familles 4 à 9 traitent autant des moyens, des objectifs et des résultats d'un PIHT alors que les autres familles sont concentrées soit sur le couple Moyens/Objectifs, représentatif du contrôle de processus, soit sur le couple Objectifs/Résultats, représentatif du contrôle de résultat. Ce recouvrement observé entre les stratégies «processus » et « résultat » pour les six familles de MC identifiées du $\mathrm{n}^{\circ} 4$ au $\mathrm{n}^{\circ} 9$ nous permet d'introduire une nouvelle dimension intermédiaire entre processus et résultat. 
De fait, aux deux stratégies précédemment identifiées, une troisième doit être ajoutée. Ces familles de MC reflètent un pragmatisme permanent qui passe par des allers et retours systématiques entre objectifs définis pour les acteurs du projet, les moyens qui leur sont alloués et les résultats obtenus. Il s'agit d'une dimension particulière, la prise de décision, selon nous représentative de la fonction des «chefs » de projet. Nous proposons donc de compléter le cœur du cadre d'analyse des moyens possibles de contrôle de PIHT avec une stratégie dénommée «arbitrage ». Il s'agit de l'un des points originaux de notre travail si on le compare à la littérature «classique ».

Sachant que nous présentons l'intégralité des moyens de contrôle de PIHT possibles en annexe 1, nous pouvons maintenant résumer dans le tableau suivant l'ensemble des familles de MC associées à chacune des trois stratégies définies : 


\begin{tabular}{|c|l|c|}
\hline Stratégie & \multicolumn{1}{|c|}{ Familles de Moyens de Contrôle } & Nombre de Moyens de contrôle associés \\
\hline \multirow{5}{*}{ PROCESSUS } & Favoriser la culture innovatrice des acteurs de l'entreprise & 9 \\
\cline { 2 - 3 } & Contenir la différenciation des fonctions de l'entreprise & 9 \\
\cline { 2 - 3 } & Rechercher l'intégration des fonctions de l'entreprise & 17 \\
\hline & Sous-total Processus & $\mathbf{3 5}$ \\
\hline \multirow{5}{*}{ ARBITRAGE } & Gérer le slack & 5 \\
\cline { 2 - 3 } & Piloter la qualité au sein de l'entreprise en concevant de façon pragmatique & 4 \\
\cline { 2 - 3 } & Evaluer le projet d'innovation de haute technologie & 7 \\
\cline { 2 - 3 } & Piloter l'allocation des ressources aux tâches & 5 \\
\cline { 2 - 3 } & Piloter les coûts de projet & 10 \\
\cline { 2 - 3 } & Arbitrer entre des dimensions Coûts/Délais/Qualités & 5 \\
\hline \multirow{3}{*}{ RESULTAT } & Sous-total Arbitrage & $\mathbf{3 6}$ \\
\cline { 2 - 3 } & Piloter les délais du projet & 3 \\
\cline { 2 - 3 } & Piloter la qualité perçue du produit ou service par un client externe à l'entreprise & 7 \\
\cline { 2 - 3 } & Piloter le chiffre d'affaires prévisionnel du produit ou service développé & 2 \\
\cline { 2 - 3 } & Piloter les coûts de l'objet développé & 5 \\
\hline & Sous-total Résultat & $\mathbf{1 7}$ \\
\hline & Total & $\mathbf{8 8}$ \\
\hline
\end{tabular}

Tableau 3 : présentation synthétique du cadre d'analyse du contrôle de PIHT 
A partir de cette grille d'analyse, qui constitue le premier apport de notre recherche, nous pouvons maintenant repérer, sur quatre études de cas, les comportements spécifiques de chefs de projets.

\section{METHODOLOGIE ET ETUDES DE CAS}

\subsection{LE PROGRAMME DE RECHERCHE REALISE AU SEIN DE FRANCOPOLE}

Avec Francopole, un opérateur majeur du secteur des télécommunications, nous avons eu l'opportunité de mener une recherche-intervention longitudinale (David et al. 2000, 102) sur des projets d'innovation technologique. Celle-ci court de 1995 à 1998. Durant cette période, le chercheur a été rattaché à une direction du contrôle de gestion et non à des départements de R\&D, d'ingénierie ou de marketing, où se situent la majorité des acteurs des projets observés. A l'usage, cela s'est révélé être un atout : nous faisons partie de l'organisation de Francopole sans avoir de poids hiérarchique sur ces acteurs opérationnels de PIHT. Le chercheur est dans une position d'observateur, les acteurs rencontrés étant conscients d'être sous observation.

A chaque entretien ou présentation du projet de recherche, le double statut de chercheur et de contrôleur de gestion a été brièvement présenté à chaque interlocuteur : les acteurs rencontrés sont le plus souvent d'avis que ce double statut offre à la fois une certaine proximité et une certaine distance par rapport à leurs préoccupations.

Parmi toutes les postures possibles définies par Vinten (1987), du plus immergé («participant à part entière ») au moins impliqué («observateur à part entière »), notre posture est bien celle

d'un «observateur participant». Nous en rappelons les caractéristiques dans le tableau cidessous : 


\begin{tabular}{|c|c|c|c|c|}
\hline Thème & $\begin{array}{l}\text { Participant à } \\
\text { part entière }\end{array}$ & $\begin{array}{l}\text { Participant } \\
\text { observateur }\end{array}$ & $\begin{array}{l}\text { Observateur } \\
\text { participant }\end{array}$ & $\begin{array}{l}\text { Observateur à } \\
\text { part entière }\end{array}$ \\
\hline $\begin{array}{l}\text { Appartenance } \\
\text { au groupe }\end{array}$ & $\begin{array}{l}\text { Membre du } \\
\text { groupe à part } \\
\text { entière }\end{array}$ & $\begin{array}{l}\text { Membre du } \\
\text { groupe }\end{array}$ & $\begin{array}{l}\text { Acteur } \\
\text { interagissant } \\
\text { avec le groupe } \\
\end{array}$ & $\begin{array}{l}\text { Sujets non } \\
\text { prévenus de } \\
\text { l'observation } \\
\end{array}$ \\
\hline $\begin{array}{l}\text { Niveau de détail } \\
\text { des données }\end{array}$ & Fort & Assez fort & Assez faible & Faible \\
\hline Risque éthique & Assez faible & Assez faible & Assez faible & Forte \\
\hline $\begin{array}{l}\text { Effet } \\
\text { d'Hawthorne }\end{array}$ & Faible & Fort & Fort & Faible \\
\hline
\end{tabular}

Tableau 4 Vinten (1987) : caractéristiques de la méthode d'observation retenue

Le principal biais de cette posture - l'effet d'Hawthorne - a été pris en compte et compensé par une observation longitudinale sur près de trois années. Le délai moyen de quatre mois entre deux entretiens a notamment permis de mettre à l'épreuve du temps la cohérence entre le discours et les préoccupations des chefs de projet.

\subsection{SIX PROJETS RETENUS INTIALEMENT, QUATRE FINALEMENT OBSERVES}

Les projets retenus cherchent tous à aboutir à une offre commerciale dans un délai d'environ deux ans. Ils peuvent tous être considérés comme visant une innovation fondée sur de la haute technologie même s'il est à noter qu'aucun ne porte une innovation radicale au sens de Zaltman \& al. (1973) : ils sont de fait tous porteurs d'une innovation plutôt progressive. Ils peuvent également tous être considérés comme de type $\mathrm{C}$, à savoir relativement petits et internes à l'entreprise Francopole. A titre d'exemple, leurs principales charges directes représentent environ vingt-cinq hommes-an par projet au total. Enfin ces quatre projets sont compatibles et indépendants, ce qui limite leurs interactions.

Nous avons pu observer une dizaine de ces projets dans un premier temps. Six sont autant d'objets de recherche potentiels, leur chef de projet étant volontaire pour un travail d'analyse prolongé dans le temps. Nous présentons leurs principales caractéristiques dans le tableau 5 ci-dessous : 


\begin{tabular}{|c|c|c|c|c|c|}
\hline \multirow[t]{2}{*}{ Projet } & \multirow[t]{2}{*}{ Produit ou service innovant } & \multirow[t]{2}{*}{ Marché visé } & \multirow[t]{2}{*}{$\begin{array}{l}\text { Sens de } \\
\text { l'innovation }\end{array}$} & \multicolumn{2}{|c|}{$\begin{array}{l}\text { Aboutissement } \\
\text { commercial }\end{array}$} \\
\hline & & & & \begin{tabular}{|l} 
à \\
l'issue \\
du \\
projet
\end{tabular} & $\begin{array}{l}3 \text { ans } \\
\text { après } \\
\text { l'issue }\end{array}$ \\
\hline $\mathbf{A}$ & Répertoire et composeur vocal & Grand public & $\begin{array}{l}\text { Poussé par la } \\
\text { technologie }\end{array}$ & Non & Oui \\
\hline B & Fédération de messageries & Grand public & \begin{tabular}{|l|}
$\begin{array}{l}\text { Poussé par la } \\
\text { technologie }\end{array}$ \\
\end{tabular} & Non & Non \\
\hline $\mathbf{C}$ & $\begin{array}{l}\text { Gestion de téléphonie privée (PABX } \\
\text { virtuel) }\end{array}$ & \begin{tabular}{|l|} 
Entreprises \\
Professionnels
\end{tabular} & Tiré par le marché & Oui & Oui \\
\hline D & Service Confort & Grand public & Tiré par le marché & Oui & Oui \\
\hline $\mathbf{E}$ & Service d'information & Grand public & Tiré par le marché & Non & NA \\
\hline $\mathbf{F}$ & \begin{tabular}{|l} 
Service de maintenance \\
d'équipements en site client
\end{tabular} & \begin{tabular}{|l|} 
Entreprises \\
Professionnels \\
\end{tabular} & $\begin{array}{l}\text { Poussé par la } \\
\text { technologie }\end{array}$ & Non & NA \\
\hline
\end{tabular}

Tableau 5 : principales caractéristiques différenciant les six projets

Contrairement à nos attentes initiales, les deux projets $\mathrm{E}$ et $\mathrm{F}$ n'ont pas pu être observés sur leur durée. Le projet E visait des services spécifiques rendus à l'aide d'une architecture technique basée sur la technologie Vidéotexte. Mais cette technologie s'est avérée être trop avancée dans son cycle de vie par rapport à la technologie émergente, la technologie de transmission IP («Internet Protocol »); dès la fin du premier semestre 1996, après trois entretiens seulement, la décision était prise d'abandonner l'observation de ce projet. Quant au projet F, il devait initialement porter sur une offre de service à valeur ajoutée de maintenance d'équipements de télécommunications installés chez des clients dans le segment «professionnels ». Au moment de son lancement effectif, en août 1996, ses objectifs ont cependant été réduits à une réorganisation interne des départements de Service Après Vente, sans impact commercial innovant vu du segment de clientèle concerné : le projet est de ce fait sorti du champ de notre recherche. 
De façon plus qualitative et pour conclure, nous reprenons ci-après une phrase de chaque chef de projet permettant de caractériser son ambition, l'enjeu principal du projet dont il a la charge :

\begin{tabular}{|c|c|}
\hline Projet & Enjeu principal \\
\hline $\mathrm{A}$ & $\begin{array}{l}\text { "il y a un marché pour la reconnaissance vocale, on peut être juste avant } \\
\text { la concurrence! » }\end{array}$ \\
\hline $\mathrm{B}$ & $\begin{array}{l}\text { "il ne faut pas se rédimer (se restreindre dans un patois lyonnais) pour un } \\
\text { tel service partageable entre téléphone fixe et portable » }\end{array}$ \\
\hline $\mathrm{C}$ & $\begin{array}{l}\text { "nous pouvons faire plus que la concurrence en convaincant les } \\
\text { entreprises qui externalisent en ce moment leurs télécommunications que } \\
\text { notre service est un service de référence» }\end{array}$ \\
\hline $\mathrm{D}$ & $\begin{array}{l}\text { "ce service doit devenir d'un usage aussi simple que de décrocher son } \\
\text { téléphone » }\end{array}$ \\
\hline
\end{tabular}

Tableau 6 Enjeu majeur de leur PIHT selon chacun des chefs de projet

\subsection{LES DESCRIPTIONS DES CAS EXPLOITEES A L'AIDE D'UNE ANALYSE DE CONTENU}

Nous avons mené des entretiens avec les chefs de projet tout au long du déroulement des projets - un tous les quatre mois en moyenne - et récolté tout document pertinent à la compréhension du contrôle du projet (compte-rendu de réunion, cahier des charges, planning...). Nous avons ainsi pu recourir à «l'analyse de contenu » pour traiter de la façon la plus systématique possible ces données qualitatives. La méthode est celle décrite par Bardin (1996, pp. 89-98). Les différentes étapes de notre démarche méthodologique sont :

- Etape 1 : entretiens et collectes de documentation, repérage des Moyens de Contrôle mis en œuvre

- Etape 2 : élaboration de statistiques par projet des Moyens de Contrôle observés

Etape 2.1 : rédaction d'une synthèse par source documentaire :

Dans la semaine qui suit une collecte de source documentaire (entretien semi directif ou document de projet), nous rédigeons un premier document, une prise de note brute, dont l'objectif est de traduire les notes en termes fidèles : le verbatim y est noté en lettres italiques. 
Dans la deuxième semaine suivant l'entretien ou la réception du document, nous travaillons de nouveau sur ce document pour en préparer une synthèse. C'est à cette occasion que nous regroupons par thèmes les différentes citations ou notes de l'entretien ou du document et proposons des interprétations intermédiaires des enjeux de contrôle du chef de projet.

Etape 2.2 : analyse de contenu par repérage des Moyens de Contrôle à chaque observation :

Ce n'est qu'une fois cette synthèse réalisée pour environ la moitié des sources documentaires que le repérage des Moyens de Contrôle commence. Comme le recommande Bardin (1996), à chaque fois qu'un Moyen de Contrôle est mentionné, il s'agit d'un « indice » qui signifie que ce Moyen de Contrôle est considéré comme pertinent par le chef de projet. Ce MC n'est pas alors nécessairement mis en œuvre mais le chef de projet se pose a minima la question de son utilisation dans le cadre du projet.

Pour illustrer ce repérage des Moyens de Contrôle, nous montrons ci-dessous un exemple de prise de note pour le projet $\mathrm{C}$ et de repérage d'un moyen de contrôle lié au résultat d'un PIHT :

«(...) Les études de marché sont menées en parallèle par les gens du marketing, surtout pour situer le produit C par rapport aux services déjà commercialisés par Francopole. (...) » \begin{tabular}{|l|l}
\hline Identifiant de & Source documentaire $\mathrm{C}^{4}$
\end{tabular} l'observation $n^{\circ} 12^{3}$ \begin{tabular}{|l|l}
\hline Moyen de contrôle & Résultat / Positionner le produit ou service développé par rapport à
\end{tabular} observé : ceux offerts par l'entreprise

Tableau 7 Préparation de l'analyse de contenu : exemple de repérage d'un moyen de contrôle à partir d'un verbatim d'entretien

Il s'agit là d'une observation à un moment donné du recours à un Moyen de Contrôle possible du résultat de PIHT, en l'occurrence le $n^{\circ} 6$ de la famille «11) Piloter la qualité perçue du produit ou service par un client externe à l'entreprise » (cf. annexe 1).

\footnotetext{
${ }^{3}$ Cet identifiant est un identifiant unique non signifiant de l'observation.

${ }^{4} \mathrm{C} 5$ fait référence au $3{ }^{\text {ème }}$ entretien passé avec le chef de projet $\mathrm{C}$.
} 
L'ensemble de ces observations est repris par projet sous forme synthétique en annexe 2. Le dénombrement total est de 725 Moyens de Contrôle observés longitudinalement sur ces quatre projets.

Il est à noter qu'il ne s'agit pas nécessairement de Moyens de Contrôle différents ; il est par exemple tout à fait possible qu'un chef de projet recoure plusieurs fois à un même MC dans le déroulement du projet. En l'occurrence pour le projet C, dans les 24 observations de MC de la famille «11) Piloter la qualité perçue du produit ou service par un client externe à l'entreprise », une autre observation a pu être faite du même $\mathrm{MC}$, mais plus tardivement, à savoir au moment de l'entretien C13, plus d'une année après la première observation.

Pour archiver ces différentes informations, nous nous appuyons sur une base de données qui recense d'une part toutes les observations sous la forme de texte court avec identifiant unique et d'autre part tous les MC recensés dans la synthèse de la littérature ${ }^{5}$. Cette base de données est un support pour mémoriser la façon dont nous qualifions les comportements de chef de projet en MC de PIHT.

- Etape 3 : comparaison par projets

- Etape 4 : analyse et écriture des résultats

\section{COMMENT UN CHEF DE PROJET PEUT-IL CONTROLER UN PROJET D'INNOVATION DE HAUTE TECHNOLOGIE?}

Nous cherchons, dans cette partie, à répondre aux trois questions suivantes qui déclinent notre question de recherche générale :

\footnotetext{
${ }^{5}$ Cette base gérée sous Microsoft Access ${ }^{\circledR}$ contient deux tables principales : celle des moyens de contrôle possibles (revue de littérature) et celles des cas (moyens de contrôle observés). La table des cas porte la référence à la phase de gestion du projet. Le modèle mis en place s'appuie sur une intégrité inférentielle et une relation « un à plusieurs » entre la clé de la table des moyens de contrôle possibles et la clé de la table des cas.
} 
- quantitativement : faut-il recourir à tous les moyens possibles de contrôle ?

- qualitativement : faut-il le faire en se focalisant sur certains ?

- dans le temps : faut-il donner la priorité à une stratégie de contrôle particulière lors de chacune des phases de PIHT : avant-projet, de réalisation et évaluation ?

Nos réponses sont bien entendu à considérer comme des « propositions » transitoires.

\subsection{LE RECOURS A L'ENSEMBLE DES FAMILLES DE MOYENS DE CONTROLE}

Nos observations (cf. annexe 2) montrent que les chefs de projet observés dans les quatre cas suivis longitudinalement à Francopole recourent à au moins un moyen de contrôle de chacune des treize familles de Moyens de Contrôle de PIHT proposés dans le cadre d'analyse (cf. tableau 3). Que le projet aboutisse ou non à un produit ou à un service commercialisé, ces quatre projets ont en effet été menés à leur terme, au bout de trois années de travail. Recourir à l'ensemble de ces Moyens de Contrôle semble être une condition nécessaire d'un PIHT, notamment pour couvrir les trois stratégies de contrôle que sont le processus, l'arbitrage et le résultat. Nous formulons ainsi la proposition suivante :

Proposition 1 : pour contrôler un PIHT, un chef de projet recourt au moins à un moyen de contrôle de chacune des treize familles possibles de Moyens de Contrôle de PIHT recensées dans le cadre d'analyse.

Mais l'utilisation de ces différents MC n'apparaît pas discriminante pour différencier les différents projets. Il nous faut, en suivant Simons, analyser comment les chefs de projet utilisent ces MC. L'intensité de l'utilisation de ces Moyens de Contrôle permet-elle alors de distinguer les différents projets? Cela nous permettrait de qualifier l'éventuelle utilisation interactive des systèmes de contrôle par les chefs de projet. 


\subsection{L'INTENSITE DU RECOURS AUX DIFFERENTS MOYENS DE CONTROLE}

Pour déterminer si les chefs de projet utilisent de manière interactive ou non les systèmes de contrôle à leur disposition, nous nous intéressons à l'intensité du recours à certains des MC dont ils disposent. Une interactivité élevée doit se traduire par une intensité relativement forte dans l'utilisation de quelques MC jugés plus efficaces. Cela signifie que les managers ne dispersent pas leur attention sur un trop grand nombre de MC. A défaut de pouvoir mesurer directement l'intensité, nous mesurons la dispersion de l'attention managériale (qui traduit une faible intensité). Nous définissons ainsi « la dispersion du contrôle » comme le rapport entre le nombre de MC utilisés au moins une fois par un projet donné et le nombre de Moyens de Contrôle potentiellement associés à la même famille de Moyens de Contrôle. Cette valeur est un pourcentage inférieur ou égal à $100 \%$. Une valeur faible de dispersion traduit une grande intensité de l'attention managériale sur des MC disponibles et un recours plus interactif aux MC utilisés.

Par exemple, nous observons que le chef de projet A recourt au moins une fois aux Moyens de Contrôle suivants parmi tous les MC possibles pour la famille «Rechercher l'intégration des fonctions de l'entreprise » : 


\begin{tabular}{|c|c|c|c|c|}
\hline Stratégie & $\begin{array}{l}\text { Famille } \\
\text { associée }\end{array}$ & MC associé & Utilisé & Calcul \\
\hline \multirow[t]{18}{*}{ Processus } & \multirow{16}{*}{$\begin{array}{l}\text { 3) Rechercher } \\
\text { l'intégration } \\
\text { des fonctions } \\
\text { de l'entreprise }\end{array}$} & $\begin{array}{l}\text { 1) Adapter les contraintes temporelles des différentes } \\
\text { fonctions au projet }\end{array}$ & Oui & 1 \\
\hline & & $\begin{array}{l}\text { 2) Affecter les sous budgets de façon formelle par } \\
\text { domaine de responsabilité entre le projet et les } \\
\text { fonctions }\end{array}$ & Non & 0 \\
\hline & & $\begin{array}{l}\text { 3) Communiquer par rapport formel en cas de } \\
\text { problème nouveau seulement }\end{array}$ & Non & 0 \\
\hline & & $\begin{array}{llll}4) \text { Communiquer systématiquement de façon } \\
\text { informelle }\end{array}$ & Non & 0 \\
\hline & & $\begin{array}{l}\text { 5) Confier la maîtrise d'ouvrage projet à des clients } \\
\text { internes de l'innovation }\end{array}$ & Oui & 1 \\
\hline & & $\begin{array}{l}\text { 6) Créer des relations client/fournisseur avec une } \\
\text { contractualisation entre maîtrise d'ouvrage et de } \\
\text { maîtrise d'œuvre, traduite soit dans un document } \\
\text { technico-économique succinct soit dans un cahier des } \\
\text { charges complet }\end{array}$ & Oui & 1 \\
\hline & & $\begin{array}{l}\text { 7) Faire preuve de volonté pour faire prendre des } \\
\text { risques au sein de l'entreprise }\end{array}$ & oui & 1 \\
\hline & & $\begin{array}{l}\text { 8) Fixer des objectifs Maîtrise d'ouvrage - Maîtrise } \\
\text { d'Euvre à l'aide de scénarios }\end{array}$ & Oui & 1 \\
\hline & & $\begin{array}{l}\text { 9) Formaliser les responsabilités en cas de mécanisme } \\
\text { organisationnel transverse en recourant à une } \\
\text { terminologie commune }\end{array}$ & Non & 0 \\
\hline & & $\begin{array}{l}\text { 10) Intéresser les autres fonctions impliquées en } \\
\text { maximisant l'impact favorable du projet d'innovation } \\
\text { sur leurs objectifs }\end{array}$ & Oui & 1 \\
\hline & & $\begin{array}{l}\text { 11) Mettre en place des contacts directs entre } \\
\text { fonctions }\end{array}$ & Oui & 1 \\
\hline & & $\begin{array}{l}\text { 12) Mettre en place des groupes de travail entre } \\
\text { fonctions }\end{array}$ & Oui & 1 \\
\hline & & $\begin{array}{l}\text { 13) Mettre en place une fonction de gate-keeper } \\
\text { comme fonction de médiation }\end{array}$ & Oui & 1 \\
\hline & & $\begin{array}{l}\text { 14) Recourir aux NTIC pour faciliter les échanges } \\
\text { d'information }\end{array}$ & Oui & 1 \\
\hline & & $\begin{array}{l}\text { 15) Revoir l'avancement d'un projet par un comité de } \\
\text { suivi }\end{array}$ & Non & 0 \\
\hline & & $\begin{array}{l}\text { 16) S'aider d'un prototype ou d'un objet préexistant } \\
\text { dans l'entreprise }\end{array}$ & Non & 0 \\
\hline & & \begin{tabular}{|lcr} 
17) S'assurer du soutien d'un champion & de \\
l'innovation ayant du pouvoir dans l'organisation & \\
\end{tabular} & Oui & 1 \\
\hline & & Total & oui & 11 \\
\hline & & \begin{tabular}{|l|} 
Dispersion \\
\end{tabular} & $11 / 1$ & $65 \%$ \\
\hline
\end{tabular}

Tableau 8 : exemple de calcul de la dispersion des Moyens de Contrôle pour le projet $\mathbf{A}$ et pour la famille «Rechercher l'intégration des fonctions de l'entreprise »

Dans cet exemple, la dispersion observée est donc de 65\%. Plus généralement, une fois l'ensemble des dispersions calculées, nous observons les moyennes suivantes : 


\begin{tabular}{|l|l|l|}
\hline Projet & Dispersion & Ecart/moyenne \\
\hline A & $69 \%$ & $9 \%$ \\
\hline B & $66 \%$ & $6 \%$ \\
\hline C & $55 \%$ & $-5 \%$ \\
\hline D & $51 \%$ & $-9 \%$ \\
\hline Moyenne & $\mathbf{6 0 \%}$ & \\
\hline
\end{tabular}

Tableau 9 : dispersion moyenne des Moyens de Contrôle par cas

Deux projets ont paru se disperser relativement plus que les autres dans leur gestion des MC possibles. Le projet A (un échec commercial mais dont les résultats techniques ont été réutilisés commercialement trois ans après) recourt en moyenne à relativement plus de Moyens de Contrôle que les autres projets (69\% contre moyenne 60\%). Il se distingue en particulier de la moyenne des autres projets pour les familles de Moyens de Contrôle «Piloter le chiffre d'affaires prévisionnel du produit ou service développé » et « Evaluer le projet ». Le projet $\mathrm{B}$, également un « échec », affiche aussi une dispersion supérieure à la moyenne (66\% contre 60\%). Cet écart est particulièrement élevé pour la famille de Moyens de Contrôle «Favoriser la culture innovatrice des acteurs de l'entreprise». Les chefs de projet A et B peinent à focaliser leur attention sur quelques Moyens de Contrôle qui seraient alors utilisés de façon interactive pour améliorer les échanges entre participants au projet.

Les deux autres projets sont des succès dans la mesure où ils aboutissent à un lancement commercial réussi. En ce qui concerne le projet $\mathrm{C}$, la dispersion observée est inférieure à la moyenne (55\% contre 60\%), notamment avec les familles de Moyens de Contrôle «Favoriser la culture innovatrice des acteurs de l'entreprise ». Nous pouvons l'expliquer par le fait que le chef de projet nous paraît avant tout concentré sur la recherche de soutien de la part de sa hiérarchie pour son projet, indépendamment de son caractère innovant, qui mobilise «naturellement » les autres contributeurs ( $R \& D$, ingénierie). L’interactivité recherchée a donc 
été verticale, à destination de la hiérarchie. Le chef de projet a donc surtout recherché des sponsors internes et à mobiliser ses outils de gestion plutôt qu'il n'a géré le projet en luimême. Cette explication se retrouve dans l'observation suivante : la dispersion de la famille de $\mathrm{MC}$ «Gérer le slack» est aussi significativement inférieure à la moyenne, peut-être tout simplement parce que la marge globale déléguée au chef de projet est relativement faible.

Enfin, en ce qui concerne le projet D, la dispersion moyenne est la plus faible (51\% contre $60 \%$ en moyenne). Les deux familles de MC suivants sont ainsi observées avec une dispersion significativement en dessous de la moyenne: «Piloter les délais du projet» et «Piloter l'allocation des ressources aux tâches ». Cette différence traduit une différence de style du chef de projet qui veut définir des priorités plus marquée que ses collègues. La gestion des délais est primordiale pour ce projet en période de déréglementation des télécommunications. L’interactivité, focalisée sur quelques Moyens de Contrôle, s'exerçait alors davantage de manière horizontale (à l'instar de ce qui est décrit par Tani, 1995), c'est-à-dire pour les participants aux projets qui se sont alors trouvés très impliqués. Dans le même temps, le projet dépend des «VN » ou «Versions Numériques » bisannuelles, un déploiement groupé d'un ensemble de corrections et d'améliorations dans tous les réseaux de Francopole. Le chef de projet nous a donc semblé tout faire («piloter l'allocation des ressources aux tâches ») pour privilégier l'urgence («piloter les délais du projet») d'obtenir la décision d'inclure le déploiement des fonctionnalités des réseaux nécessaires à l'innovation D soient programmées dans l'une de ces VN, avant de marquer un certain relâchement, une fois cette décision obtenue: la mise en œuvre de ce service a ainsi bénéficié d'un style particulièrement intrapreneur du chef de projet, capable de convaincre de la nécessité d'investir dans un développement structurant à un moment clé. 
Le chef du projet $\mathrm{D}$ nous a paru plus à même de se concentrer sur quelques actions qui lui semblaient prioritaires pour le meilleur déroulement possible du projet. Nous pensons donc que le style de contrôle du projet $\mathrm{D}$ est en bonne partie lié à cette capacité de son chef de projet. Le chef de projet C s'est investi dans l'avancement technique des travaux d'innovation, principalement parce qu'il était insuffisamment soutenu par sa hiérarchie marketing. Les chefs de projet A et B ont dispersé leurs efforts de contrôle, soit pour intéresser le marketing (projet A), soit pour faire avancer les développements de la plate-forme de développement partageable par d'autres projets (projet B). Si le degré d'innovation technologique ne nous paraît pas discriminant pour les quatre projets, il semble plutôt qu' une différence qualitative existe entre le projet $\mathrm{C}$ et $\mathrm{D}$ et les deux autres : la gestion de ces projets est plus avare en MC utilisés car les chefs de projet tiennent à éviter une dispersion des efforts de contrôle.

Proposition 2 : les chefs de projet donnent d'autant plus de chances à l'innovation de haute technologie d'aboutir qu'ils dispersent moins leurs efforts de contrôle en focalisant leur attention sur certains Moyens de Contrôle. Le recours relativement intensif aux Moyens de Contrôle sert à des échanges plus interactifs et efficaces entre les différentes parties prenantes au projet.

\subsection{LA PRIORITE dONNEe AU CONTROLe de PROCESSUS PENDANT La PHASE D’AVANT-PROJET D’Un PIHT}

La phase d'avant-projet est de fait une phase de maturation d'une idée d'innovation et d'une équipe-projet pour la développer. A ce stade, l'illusion de son contrôle par un seul chiffre de valeur actuelle nette (VAN) ne devrait pas y avoir sa place: même accompagné de commentaires prudents, un chiffre unique peut faire oublier l'incertitude des hypothèses sur lesquelles il repose (Bower, 1986). Lors de la phase d'avant-projet d'un PIHT, les échéances

sont en effet à un terme trop éloigné dans le temps par rapport aux possibilités connues, 
commerciales, technologiques ou organisationnelles. Si l'enjeu est économique, c'est d'abord sous un angle stratégique et qualitatif. En reprenant la thèse de March et Shapira (1991) selon laquelle le risque se mesure avant tout par une «fourchette », les seuls chiffres souhaitables à ce stade d'un PIHT devraient être une fourchette de coût cible du produit ou service et une fourchette de dépenses du projet.

Il semble que l'avant-projet doit globalement intégrer par construction une attention plus forte aux aspects d'organisation qu'au résultat attendu, notamment financier. Les deux familles de Moyens de Contrôle d'intégration et de différenciation simultanée des différentes fonctions devraient ainsi être primordiales. L'enjeu est bien de porter un jugement sur l'innovation en gestation, que ce soit par l'équipe-projet ou par les différents décideurs impliqués. Ce temps est précieux pour forger un premier jeu d'objectifs simplement étayé par des ordres de grandeur économiques. Les acteurs des différentes fonctions se «mettent en route » vers l'innovation, démarche caractéristique des MC liés au processus de PIHT plus qu'aux deux autres stratégies de contrôle, arbitrage et résultat.

Dans les cas observés, cette tendance se confirme de façon homogène, sans qu'il soit possible de différencier les études de cas les unes des autres. Le pourcentage des MC observés par phase de gestion de PIHT et moyenné sur les quatre cas est le suivant : 


\begin{tabular}{|l|c|c|c|c|}
\hline & Avant-projet & Réalisation & Evaluation & Total \\
\hline Processus & $40 \%$ & $40 \%$ & $34 \%$ & $40 \%$ \\
\hline Arbitrage & $36 \%$ & $33 \%$ & $38 \%$ & $34 \%$ \\
\hline Résultat & $24 \%$ & $27 \%$ & $28 \%$ & $26 \%$ \\
\hline Total & $100 \%$ & $100 \%$ & $100 \%$ & $100 \%$ \\
\hline
\end{tabular}

Tableau 10 : pourcentage des stratégies de contrôle observés dans la phase d'avantprojet dans l'ensemble des quatre cas ${ }^{6}$

Nous formulons ainsi la proposition suivante :

Proposition 3 : la priorité est accordée par les chefs de projet étudiés au contrôle du processus de PIHT lors de la phase d'avant-projet des PIHT observés. Les chefs de projet construisent et affinent les critères qui vont ensuite être utilisés pour les stratégies d'arbitrage et de résultat.

\subsection{LA PRIORITE DONNEe AU CONTROLE D'ARbitrage PENDANT LA PHASE DE} REALISATION D'UN PIHT

Le dénombrement des MC observés sur les quatre cas montre les poids respectifs des trois stratégies de contrôle lors de la phase de réalisation (cf. tableau 10). La hiérarchie est comparable à celle de la phase d'avant-projet et nous observons une légère baisse relative de la stratégie arbitrage (36\% à 33\%) entre la phase d'avant-projet et la phase de réalisation. En première approche, l'arbitrage semble donc être la stratégie de contrôle la moins prioritaire selon ce critère plutôt quantitatif.

Une analyse plus qualitative montre pourtant que l'arbitrage est sans doute plus important qu'il n'y paraît. Si le nombre de Moyens de Contrôle consacrés à l'arbitrage de PIHT n'est pas

\footnotetext{
${ }^{6}$ Ce tableau présente de façon synthétique les mêmes données que celles de l'annexe 2 ; la différence avec l'annexe 2 est que celle-ci sont présentées par phase ; pour exemple, le pourcentage consacré au processus sur l'ensemble des phases est ici de $40 \%$ (total de la première ligne du tableau de l'annexe 2) ce qui correspond au calcul suivant dans l'annexe : sous-total processus $(288) /$ total $(725)=40 \%$.
} 
supérieur en valeur relative, le nombre de Moyens de Contrôle, en valeur absolue, consacrés à la stratégie « arbitrage » augmentent :

\begin{tabular}{|l|l|l|l|l|}
\hline Projet & Avant-projet & Réalisation & Evolution & Evolution \\
\hline A & 29 & 76 & +47 & $+162 \%$ \\
\hline B & 25 & 32 & +7 & $+28 \%$ \\
\hline C & 18 & 16 & -2 & $-11 \%$ \\
\hline D & 19 & 16 & -3 & $-16 \%$ \\
\hline
\end{tabular}

Tableau 11 : Evolution du nombre de MC liés à l'« arbitrage » de PIHT entre les phases d'avant-projet et de réalisation

De fait, les évolutions sont contrastées entre les projets A et B d'une part, C et D d'autre part. Repartant du constat fait plus haut (cf. proposition 2), nous pensons que les projets ayant abouti commercialement $(\mathrm{C}$ et $\mathrm{D})$ dispersent moins leurs efforts de contrôle. A l'inverse, le chef de projet $\mathrm{A}$ - dans une moindre mesure $\mathrm{B}$ - fait «feu de tout bois » notamment pour intéresser différents acteurs au développement de son service innovant. Cependant, l'usage extensif de plus nombreux MC traduit vraisemblablement le caractère moins décisif du contrôle qu'il exerce. Nous supposons ici que cette évolution traduit la recherche d'un accord «politique » plus clair entre les différentes fonctions R\&D, marketing et ingénierie autour de l'innovation plutôt que l'avancement concret de cette innovation. Nous pouvons donc en tirer une proposition plus qualitative sur la priorité du contrôle pendant la phase de réalisation, cette phase charnière où les acteurs sortent progressivement de la période de tâtonnements collectifs propre à la période d'avant-projet. Avec une approche différente, ce résultat vient conforter les observations de Gautier et Lenfle (2004) selon lesquels les critères utilisés pour évaluer les phases de réalisation sont construits dans les phases amont, en particulier les critères et les priorités qui permettent de rendre des arbitrages. Nous le formulons ainsi : 
Proposition 4 : le nombre de Moyens de Contrôle consacrés à l'arbitrage reste également en nombre restreint dans les projets qui aboutissent afin de permettre une interaction de qualité entre les acteurs intéressés à un projet.

\subsection{Le CAS DE LA PHASE D'EVAluation D'Un PIHT}

Une innovation amenée sur le marché, donc un succès, est caractérisée par différentes familles de MC liées au résultat: il s'agit d'un produit ou service perçu comme innovant par des clients (famille «Piloter la qualité perçue du produit ou service par un client externe à l'entreprise »), qui vont l'acheter à l'entreprise (famille "Piloter le chiffre d'affaires prévisionnel du produit ou service développé »), en offrant une certaine rentabilité à celle-ci (famille «Piloter les coûts de l'objet développé»), et ce juste avant les concurrents (famille «Piloter les délais du projet »).

Cependant, si le projet n'aboutit pas, s'il est arrêté en cours de route ou s'il est perçu comme un échec (absence de commercialisation de l'innovation ou résultats de ventes jugés insuffisants), que devient cette phase de gestion ? Par analogie avec le rôle des managers donnant du sens à la relation entre les indicateurs financiers et les indicateurs opérationnels, l'équipe-projet doit, dans cette phase d'évaluation, pouvoir faire comprendre les causes de l'échec du projet et favoriser l'apprentissage de l'organisation. Dans le cas particulier d'un échec commercial il est souhaitable de concentrer les forces d'évaluation sur le processus du PIHT. Garder en mémoire un projet en échec peut paradoxalement être un résultat.

En l'occurrence, les quatre projets observés longitudinalement ont bien été menés à leur terme. Les projets $\mathrm{A}$ et $\mathrm{B}$ n'ont pas abouti immédiatement à la commercialisation attendue : leur développement sur «étagère »a été réutilisé environ trois années après la fin du projet 
dans un autre service commercialisé par l'entreprise. Les projets $C$ et $D$ ont en revanche abouti à la commercialisation espérée.

Toutefois, nous ne disposons d'observations strictement liées à la phase d'évaluation que pour le projet C. Il s'agit de quarante-sept Moyens de Contrôle parmi les cent cinquante-trois observés en totalité pour ce cas. Même si nous ne disposons pas d'autant d'observations que pour nos conclusions précédentes, le retour d'expérience des chefs de projet nous permet malgré tout de formuler la proposition suivante :

Proposition 5 : lors de la phase d'évaluation, le contrôle d'un PIHT considéré comme une réussite commerciale semble donner la priorité au résultat. Le contrôle d'un PIHT considéré comme un échec commercial semble donner la priorité au processus. Dans le premier cas, l'interactivité n'est plus indispensable au projet. Seul un contrôle diagnostique subsiste. Dans le second, l'interactivité continue, grâce à des MC orientés vers la compréhension de l'échec afin de capitaliser pour les prochains PIHT.

Nous représentons ces propositions par la figure suivante : 


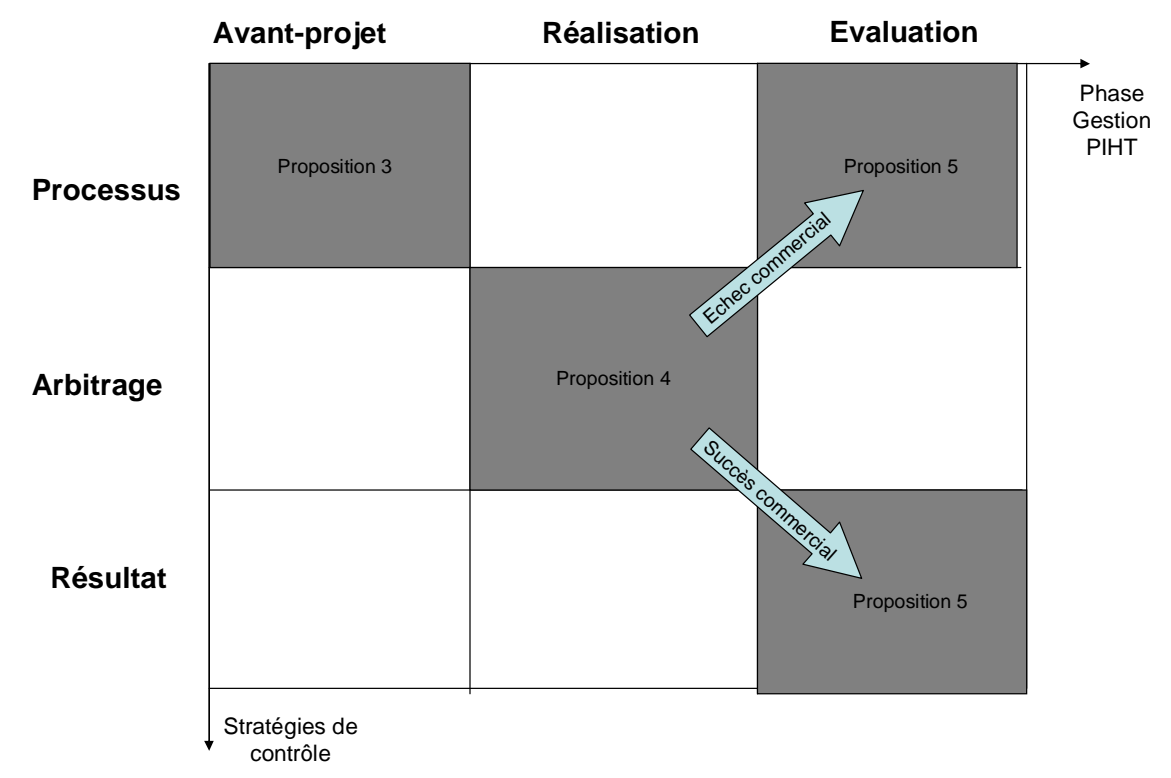

\section{Figure 3 : Représentation des trois propositions liées au temps}

Nous retrouvons en partie l'un des résultats de Nixon (1998) pour qui, au fur et à mesure que le projet avance, les mesures deviennent de plus en plus précises, plus centrées sur des éléments techniques ou financiers, plus formelles et explicites. Cela n'est vrai dans notre étude que pour les projets qui sont des succès.

Les cases grisées de la figure précédente montrent les situations critiques. En phase d'avantprojet, il est vital de s'assurer que les acteurs veulent travailler ensemble. L'ensemble des Moyens de Contrôle porte donc prioritairement sur le contrôle des proces sus. Un certain flou est accepté en ce qui concerne les stratégies de contrôle arbitrage et résultat, flou qui permet sans doute même aux projets d'avancer en ne spécifiant pas à l'excès les attendus. Dans la phase de réalisation, la stratégie «arbitrage»devient importante, plus qualitativement que quantitativement. Il s'agit pour les chefs de projets de prendre ou de faire prendre des décisions, de trancher entre des alternatives et d'engager le futur de façon plus ou moins irrémédiable. Dans la phase d'évaluation, les choses se compliquent. Si le projet est un échec 
commercial, les acteurs reviennent alors prioritairement à la stratégie «processus » qui permet d'engranger de l'information pour les prochains projets. On analyse alors l'échec afin de mieux préparer le futur. Si le projet est un succès commercial en revanche, il s'agit de montrer aux acteurs externes du projet, les bons résultats du projet. Cela permet de légitimer le projet et de renforcer le message vis-à-vis des commerciaux afin qu'ils s'investissent encore mieux dans les projets qui sont en maturation.

\section{CONCLUSION}

L'apport de cet article est double. Le premier apport est d'ordre théorique. Une grille de lecture issue de la littérature est proposée d'afin d'analyser la façon dont sont contrôlés les projets étudiés, les PIHT. Cette grille d'analyse se singularise par la mise en évidence d'une nouvelle stratégie de contrôle, l'«arbitrage », quand la plupart des auteurs distinguent «pilotage des processus » et «pilotage des résultats ». Il s'agit d'une dimension typique de la responsabilité d'intrapreneur déléguée par une entreprise au chef de projet et à l'équipe projet.

Le deuxième apport renvoie directement à l'exploitation de nos données empiriques. Il apparaît en effet à l'examen de nos données que l'interactivité mise en œuvre par les chefs de projet varie. Les plus performants semblent éviter la dispersion et se concentrent sur quelques Moyens de Contrôle quand les responsables des projets qui connaissent un échec de commercialisation se dispersent plus. Ils manquent ainsi l'objectif d'apprentissage qui peut être assigné aux MC d'un PIHT. Par ailleurs, dans la phase d'avant-projet, les chefs de projet sont davantage centrés sur les processus que sur toute autre stratégie de contrôle. Dans la phase de réalisation, ils semblent se concentrer sur l'arbitrage tout en restant focalisés, pour les plus performants, sur quelques Moyens de Contrôle. Dans la phase d'évaluation, la 
situation semble dépendre de l'issue du projet. Si c'est un échec de commercialisation, les Moyens de Contrôle utilisés sont focalisés sur les processus afin de favoriser l'apprentissage organisationnel. Si le projet est un succès, les Moyens de Contrôle utilisés sont concentrés sur l'affichage des résultats.

Cette analyse a toutefois été réalisée à partir d'un nombre réduit d'observations. Aussi est-il important dans une prochaine phase de vérifier ces résultats à partir d'un nombre de cas plus important. De même, des prolongements théoriques nous sont suggérés par les résultats obtenus. Il s'agirait en particulier de confronter ces résultats aux conclusions de Bisbe \& Otley (2004) qui indiquent qu'un contrôle interactif favoriserait globalement l'innovation-produit dans les entreprises peu innovantes, à l'inverse des entreprises très innovantes. Cette réflexion porterait ainsi plus sur le contrôle d'un portefeuille de projets plutôt que sur le contrôle d'un projet spécifique.

\section{Bibliographie}

Abernethy M. A. et Brownell P. (1999), « The role of budgets in organizations facing strategic change: an exploratory study », Accounting, Organizations and Society, vol. 24, n³, pp. 189204.

Akrich M., Callon M. et Latour B. (1988), «A quoi tient le succès des innovations?», Annales des Mines - Gérer et comprendre, juin et septembre, pp. 4-17 et 14-29.

Alter N. (1995), «Peut-on programmer l'innovation? », Revue Française de Gestion, MarsAvril-Mai, pp. 78-86.

Alter N. (1998), "Organisation et innovation, une rencontre conflictuelle », Sciences Humaines $\mathrm{n}^{\circ}$ 20, Hors Série, pp. 185-192.

Anthony R. N. (1988), Management control of projects, Boundaries of management control, pp. 101-120, Harvard Business School Press.

Anthony R. N., Dearden J., Govindarajan V. (1992), Management control of projects: 4 case studies, Chapter 17 of Management Control Systems, 7th Edition. Homewood Illinois: Richard D. Irwin, Inc.

Bardin L. (1996), L'analyse de contenu, Presses Universitaires de France, Paris.

Bisbe J. et Otley D. (2004), «The effects of the interactive use of management control systems on product innovation », Accounting, Organizations and Society, vol. 29, n 8, pp. 709-737. 
Bouquin H. (1994), Strategy, Management Accounting and Control: beyond linear causality, Université Paris IX Dauphine, Crefige 9467.

Bower J. L. (1986), Managing the resource allocation process: a study of corporate planning and investment, Harvard Business School Press.

Bruining H., Bonnet M. et Wright M. (2004), « Management control systems and strategy change in buyouts », Management Accounting Research, vol. 15, n 2, p. 155-177.

Chiapello E. (1996), «Les typologies des modes de contrôle et leurs facteurs de contingence : un essai d'organisation de la littérature », Comptabilité - Contrôle - Audit, vol. 2, n 2, pp. 5174.

Clark K. B. et Wheelwright S. C. (1992), «Creating project plans to focus product development », Harvard Business Review, mars-avril, 70-82.

Cohendet P. Jacot J.-H. et Lorino P. (ed.), (1996), Cohérence, pertinence et évaluation, Economica, ECOSIP.

Courpasson D. et Gaillard J-M., (1991), «La collaboration R\&D-marketing : une approche empirique de la notion d'équipe-projet », Gestion 2000, n5, pp. 67-91.

Danila N. (1985), Pratique française des stratégies technologiques : bilan de l'utilisation des méthodes d'évaluation et de sélection de projets de recherche, Institut de Management Public et Fondation Nationale de la Gestion des Entreprises.

David A., Hatchuel, A. et Laufer, R. (2000), Les nouvelles fondations des sciences de gestion, Vuibert, Série Fnege.

Davila T. (2000), «An empirical study on the drivers of management control systems' design in new product development », Accounting, Organizations and Society, vol. 25, n4-5, pp.383-409.

Galbraith J. K. (1977), Organizational design, Reading Mass, Addison-Wesley.

Garel G. (2003), Le management de projet, Repères, Editions La Découverte.

Garel G., Giard V. et Midler C. (2004), Faire de la recherche en management de projet, Vuibert, Collection Fnege.

Gautier F. (2002), Les systèmes de contrôle de gestion des projets de conception et de développement de produits nouveaux: Une analyse empirique, Congrès des IAE, IAE de Paris: 23 p.

Gautier F. (2004), «Pilotage économique de 1'avant-projet: les résultats des principales recherches empiriques » in Garel G., Giard V. et Midler C., Faire de la recherche en management de projet, Vuibert, Collection Fnege.

Gautier F. et Lenfle S. (2004), «L'avant-projet : définition et enjeux » in Garel G., Giard V. et Midler C., Faire de la recherche en management de projet, Vuibert, Collection Fnege.

Giard V. et Midler C. (ed.), (1993), Pilotages de projet et entreprises, Economica, ECOSIP.

Hofstede G. (1981), «Management control of public and not-for-profit activities », Accounting, Organization \& Society, vol. 6, n³, pp. 193-211.

Latour B. (1987), Science in action, Harvard University Press, Cambridge, MA. 
Lenfle S. (2004), «Peut-on gérer l'innovation par projet ? in Garel G., Giard V. et Midler C., Faire de la recherche en management de projet, Vuibert, Collection Fnege.

March J. et Shapira Z (1991), «Les managers face au risque », in J.G March (1991), Systèmes d'information et prise de décision : des liens ambigus, Les Editions d'Organisation, pp. 109130.

Marginson, D. E. W. (2002), « Management control systems and their effects on strategy formation at middle-management levels: evidence from a U.K. organization », Strategic Management Journal, vol. 23, p. 1019-1031.

Nixon B. (1998) «Research and development performance measurement: a case study », Management Accounting Research, vol. 9, pp.329-355.

Osborn C. S. (1998), « Systems for sustainable organizations: emergent strategies, interactive control and semi-formal information », Journal of Management Studies, vol. 35, $\mathrm{n}^{\circ}$ 4, pp. 481-509.

Persiaux F. (2005), Le contrôle des projets d'innovation de haute technologie: une contribution à la théorie élaborée à partir de cas observés de façon longitudinale à France Telecom, thèse de l'Ecole des Hautes Etudes Commerciales (HEC), dirigé par M. Jean-Loup Ardoin.

Simons R. (1987), «Planning, control, and uncertainty: a process view », in W. J. Bruns et K. R.S. (eds.), Accounting and Management: Field Study Perspectives, Boston, MA, Harvard Business School Press, p. 339-362.

Simons R. (1990), « The role of management control systems in creating competitive advantage: New perspectives », Accounting, Organizations and Society, vol. 15, n 1-2, pp. 127-143.

Simons R. (1991), « Strategic orientation and top management attention to control systems », Strategic Management Journal, vol. 12, pp. 49-62.

Simons R. (1994), « How new top managers use control systems as levers of strategic renewal », Strategic Management Journal, vol. 15, pp. 169-189.

Simons R. (1995a), «Control in the age or empowerment », Harvard Business Review, March-April, pp. 80-88.

Simons R. (1995b), Levers of control, Boston, Harvard University Press.

Schumpeter J. A. (1955), The theory of economic development, Cambridge, Mass., Harvard University Press.

Tani T. (1995), «Interactive Control in Target Casot Management », Management Accounting Research, vol. 6, pp. 399-414.

Van de Ven A. H., Angle H. L. et Scott Poole M. (1989), Research on the management of innovation, Ballinger Publishing Company.

Vinck D. (ed.) (1991), Gestion de la recherche : nouvelles pratiques, nouveaux problèmes, De Boeck Publishing, pp. 1 - 520.

Vinten Gerald (1987), « The participant observer », Internal Auditor, December, pp. 25-27.

Zaltman G., Duncan R. et Holbek J., (1973), Innovation and organizations, John Wiley \& Sons. 


\section{Annexe 1 : Moyens de Contrôle possibles de PIHT}

Nous reportons de façon détaillée l'ensemble des Moyens de Contrôle repérés dans notre recherche :

\begin{tabular}{|c|c|c|}
\hline Stratégie & $\begin{array}{l}\text { Famille } \\
\text { associée }\end{array}$ & MC associé \\
\hline \multirow[t]{9}{*}{$\underline{\text { Processus }}$} & \multirow{9}{*}{$\begin{array}{l}\text { 1) Favoriser la } \\
\text { culture } \\
\text { innovatrice des } \\
\text { acteurs de } \\
\text { l'entreprise }\end{array}$} & 1) Chercher à mettre en œuvre des approches de motivation par le contenu \\
\hline & & $\begin{array}{l}\text { 2) Communiquer une culture de transparence aux acteurs des différentes } \\
\text { fonctions impliquées }\end{array}$ \\
\hline & & $\begin{array}{l}\text { 3) Développer l'orientation client des acteurs impliqués par l'innovation de haute } \\
\text { technologie }\end{array}$ \\
\hline & & 4) Eviter de mettre en œuvre des approches de motivation mécanique \\
\hline & & $\begin{array}{l}\text { 5) Rapprocher les innovateurs du monde de la production et/ou de la vente } \\
\text { (stages ou postes en usine et/ou marketing/vente) }\end{array}$ \\
\hline & & $\begin{array}{l}\text { 6) Recourir à des rites de gestion de projet d'innovation (exemple : présentation } \\
\text { des maquettes de produits ou services aux cadres dirigeants) }\end{array}$ \\
\hline & & 7) Revoir l'avancement d'un projet par un comité de suivi \\
\hline & & 8) S'assurer que le plaisir de l'innovation reste source de motivation \\
\hline & & $\begin{array}{l}\text { 9) Un projet a aussi pour objectif de renforcer les compétences collectives } \\
\text { d'innovation }\end{array}$ \\
\hline
\end{tabular}

\begin{tabular}{|c|c|c|}
\hline Stratégie & Famille associée & MC associé \\
\hline \multirow[t]{9}{*}{ Processus } & \multirow{9}{*}{$\begin{array}{l}\text { 2) Contenir la } \\
\text { différenciation } \\
\text { des fonctions de } \\
\text { l'entreprise }\end{array}$} & 1) Adapter le projet aux contraintes temporelles des différentes fonctions \\
\hline & & 2) Chercher à maintenir un nombre limité de factions s'opposant sur le projet \\
\hline & & $\begin{array}{l}\text { 3) Chercher à maintenir un nombre limité de participants impliqués dans le } \\
\text { système de décision }\end{array}$ \\
\hline & & $\begin{array}{l}\text { 4) Faire apparâtre au plus tôt les désaccords entre fonctions pour les résoudre } \\
\text { ou anticiper une demande d'arbitrage, par exemple à l'aide d'un graphe socio- } \\
\text { technique }\end{array}$ \\
\hline & & $\begin{array}{l}\text { 5) Reporter le moment où des fonctions se verront contraintes de prendre sous } \\
\text { leur responsabilité les risques induits par les autres fonctions impliquées dans le } \\
\text { projet }\end{array}$ \\
\hline & & $\begin{array}{l}\text { 6) Faire se dévoiler les opposants au projet d'innovation pour mieux négocier } \\
\text { avec eux l'avancement du projet }\end{array}$ \\
\hline & & $\begin{array}{l}\text { 7) Formaliser les responsabilités en cas de mécanisme organisationnel } \\
\text { transverse en recourant à une terminologie commune }\end{array}$ \\
\hline & & $\begin{array}{l}\text { 8) Intéresser les autres fonctions impliquées en minimisant l'impact défavorable } \\
\text { du projet d'innovation sur leurs objectifs et normes }\end{array}$ \\
\hline & & 9) S'assurer du soutien des cadres dirigeants de l'organisation \\
\hline
\end{tabular}

\begin{tabular}{|c|c|c|}
\hline Stratégie & $\begin{array}{l}\begin{array}{l}\text { Famille } \\
\text { associée }\end{array} \\
\end{array}$ & MC associé \\
\hline \multirow[t]{7}{*}{ Processus } & \multirow{7}{*}{$\begin{array}{l}\text { 3) } \\
\text { Rechercher } \\
\text { l'intégration } \\
\text { des fonctions } \\
\text { de } \\
\text { l'entreprise }\end{array}$} & 1) Adapter les contraintes temporelles des différentes fonctions au projet \\
\hline & & $\begin{array}{l}\text { 2) Affecter les sous budgets de façon formelle par domaine de responsabilité entre } \\
\text { le projet et les fonctions }\end{array}$ \\
\hline & & 3) Communiquer par rapport formel en cas de problème nouveau seulement \\
\hline & & 4) Communiquer systématiquement de façon informelle \\
\hline & & 5) Confier la maîtrise d'ouvrage projet à des clients internes de l'innovation \\
\hline & & $\begin{array}{l}\text { 6) Créer des relations client/fournisseur avec une contractualisation entre maitrise } \\
\text { d'ouvrage et de maîtrise d'œuvre, traduite soit dans un document technico- } \\
\text { économique succinct soit dans un cahier des charges complet }\end{array}$ \\
\hline & & aire preuve de volonté pour faire prendre des risques au sein de l'entreprise \\
\hline
\end{tabular}




\begin{tabular}{|l|l|}
\hline \multirow{5}{|}{} & 8) Fixer des objectifs Maîtrise d'ouvrage - Maîtrise d'Cuvre à l'aide de scénarios \\
\hline & $\begin{array}{l}\text { 9) Formaliser les responsabilités en cas de mécanisme organisationnel transverse } \\
\text { en recourant à une terminologie commune }\end{array}$ \\
\hline & $\begin{array}{l}\text { 10) Intéresser les autres fonctions impliquées en maximisant l'impact favorable du } \\
\text { projet d'innovation sur leurs objectifs }\end{array}$ \\
\hline 11) Mettre en place des contacts directs entre fonctions \\
\hline 12) Mettre en place des groupes de travail entre fonctions \\
\hline 13) Mettre en place une fonction de gate-keeper comme fonction de médiation \\
\hline 14) Recourir aux NTIC pour faciliter les échanges d'information \\
\hline 15) Revoir l'avancement d'un projet par un comité de suivi \\
\hline 16) S'aider d'un prototype ou d'un objet préexistant dans l'entreprise \\
\hline $\begin{array}{l}\text { 17) S'assurer du soutien d'un champion de l'innovation ayant du pouvoir dans } \\
\text { l'organisation }\end{array}$ \\
\hline
\end{tabular}

\begin{tabular}{|c|c|c|}
\hline Stratégie & $\begin{array}{l}\text { Famille } \\
\text { associée }\end{array}$ & MC associé \\
\hline \multirow[t]{5}{*}{ Arbitrage } & \multirow[t]{5}{*}{$\begin{array}{l}\text { 4) Gérer le } \\
\text { slack }\end{array}$} & $\begin{array}{l}\text { 1) Donner de la marge de manœuvre (exercer un contrôle relâché en adoptant une } \\
\text { attitude détachée ou accommodante) }\end{array}$ \\
\hline & & 2) Maintenir la marge de manœuvre en l'état \\
\hline & & $\begin{array}{l}\text { 3) Naviguer entre un contrôle strict et un contrôle relâché entre les phases de } \\
\text { gestion d'un projet }\end{array}$ \\
\hline & & $\begin{array}{l}\text { 4) Prendre de la marge de manœuvre (exercer un contrôle strict en adoptant une } \\
\text { attitude compétitive ou coopérative) }\end{array}$ \\
\hline & & 5) Recourir à une ou plusieurs revues détaillées du projet (« in depth review ») \\
\hline
\end{tabular}

\begin{tabular}{|c|c|c|}
\hline Stratégie & $\begin{array}{l}\text { Famille } \\
\text { associée }\end{array}$ & MC associé \\
\hline \multirow[t]{4}{*}{ Arbitrage } & \multirow{4}{*}{$\begin{array}{l}\text { 5) Piloter la } \\
\text { qualité au sein } \\
\text { de l'entreprise } \\
\text { en concevant } \\
\text { de façon } \\
\text { pragmatique }\end{array}$} & 1) Définir la technologie de production du produit ou service \\
\hline & & 2) Définir la technologie spécifique au produit ou service \\
\hline & & $\begin{array}{l}\text { 3) Fournir des livrables concrets : plans, spécifications, maquettes, résultats de } \\
\text { tests, prototypes }\end{array}$ \\
\hline & & 4) Piloter par jalons \\
\hline
\end{tabular}

\begin{tabular}{|c|c|c|}
\hline Stratégie & Famille associée & MC associé \\
\hline \multirow[t]{7}{*}{ Arbitrage } & \multirow{7}{*}{$\begin{array}{l}\text { 6) Evaluer le } \\
\text { projet }\end{array}$} & 1) Conduire un audit ex-post formel détaillé longtemps après la fin du projet \\
\hline & & 2) Evaluer la performance du processus d'un projet juste après la fin du projet \\
\hline & & 3) Prévoir explicitement un go/kill \\
\hline & & $\begin{array}{l}\text { 4) Recourir à une méthode d'évaluation formalisée des risques, l'AMDEC par } \\
\text { exemple }\end{array}$ \\
\hline & & 5) Revoir l'avancement d'un projet par un comité de suivi \\
\hline & & $\begin{array}{l}\text { 6) Sélectionner un projet en s'appuyant sur une méthode qualitative (consensus } \\
\text { ou liste de contrôle) }\end{array}$ \\
\hline & & 7) Sélectionner un projet en s'appuyant sur une méthode quantitative \\
\hline
\end{tabular}

\begin{tabular}{|c|c|c|}
\hline Stratégie & Famille associée & MC associé \\
\hline \multirow[t]{5}{*}{ Arbitrage } & \multirow{5}{*}{$\begin{array}{l}\text { 7) Piloter } \\
\text { l'allocation des } \\
\text { ressources aux } \\
\text { tâches }\end{array}$} & 1) Affecter les ressources aux responsables des différents lots de travail \\
\hline & & 2) Décomposer le travail en lots \\
\hline & & $\begin{array}{l}\text { 3) Ne pas changer de choix stratégique d'allocation des ressources en cours de } \\
\text { projet }\end{array}$ \\
\hline & & 4) Piloter par jalons \\
\hline & & 5) Niveler ou lisser les ressources à l'aide du PERT-charge \\
\hline
\end{tabular}

\section{\begin{tabular}{|l|l|l|}
\hline Stratégie & Famille associée & MC associé \\
\hline
\end{tabular}}




\begin{tabular}{|c|c|c|}
\hline \multirow[t]{10}{*}{ Arbitrage } & \multirow[t]{10}{*}{$\begin{array}{l}\text { 8) Piloter les } \\
\text { coûts de projet }\end{array}$} & $\begin{array}{l}\text { 1) Adapter la finesse de l'estimation des coûts à la durée du travail nécessaire } \\
\text { pour réaliser cette estimation }\end{array}$ \\
\hline & & $\begin{array}{l}\text { 2) Affecter les sous budgets de façon formelle par domaine de responsabilité } \\
\text { entre le projet et les fonctions }\end{array}$ \\
\hline & & 3) Contrôler un projet par sa rentabilité («cost-reimbursement contract») \\
\hline & & 4) Contrôler un projet par son coût (« fixed price contract ») \\
\hline & & $\begin{array}{l}\text { 5) Fixer des objectifs Maîtrise d'ouvrage - Maitrise d'EEuvre à l'aide de } \\
\text { scénarios }\end{array}$ \\
\hline & & 6) Suivre la valeur acquise (Coût Budgété du Travail A Effectuer) \\
\hline & & $\begin{array}{l}\text { 7) Suivre le budget encouru (Coût Budgété du Travail Prévu ou du Reste à } \\
\text { Faire) }\end{array}$ \\
\hline & & 8) Suivre le coût encouru (Coût Réel du Travail Effectué) \\
\hline & & $\begin{array}{l}\text { 9) Suivre les coûts directs du projet internes à l'entreprise et externes à } \\
\text { l'entreprise, par exemple à l'aide de courbes d'influences }\end{array}$ \\
\hline & & 10) Suivre les coûts indirects du projet indépendamment de ses coûts directs \\
\hline
\end{tabular}

\begin{tabular}{|l|l|l|}
\hline Stratégie & Famille associée & MC associé \\
\hline Arbitrage & $\begin{array}{l}\text { 9) Arbitrer entre des } \\
\text { dimensions }\end{array}$ & 1) Arbitrer entre coûts du projet et coûts de l'objet du projet \\
\cline { 3 - 3 } & \multirow{4}{*}{ Coûtts/Délais/Qualités } & 2) Arbitrer entre coûts et délais \\
\cline { 3 - 3 } & & 3) Arbitrer entre coûts et qualités \\
\cline { 3 - 3 } & & 4) Arbitrer entre qualité interne et qualité externe \\
\cline { 2 - 3 } & & 5) Arbitrer entre qualités et délais \\
\hline
\end{tabular}

\begin{tabular}{|l|l|l|}
\hline Stratégie & Famille associée & MC associé \\
\hline Résultat & \multirow{2}{*}{\begin{tabular}{l} 
po) Piloter les délais du \\
\multirow{n}{*}{}
\end{tabular}} & $\begin{array}{l}\text { projet } \\
\text { (time-span) }\end{array}$ \\
\cline { 3 - 3 } & & 2) Piloter par jalons \\
\cline { 3 - 3 } & & 3) Recourir au PERT-temps (Méthode du Chemin Critique \\
\hline
\end{tabular}

\begin{tabular}{|c|c|c|}
\hline Stratégie & Famille associée & MC associé \\
\hline \multirow[t]{7}{*}{ Résultat } & \multirow{7}{*}{$\begin{array}{l}\text { 11) Piloter la qualité } \\
\text { perçue du produit ou } \\
\text { service par un client } \\
\text { externe à l'entreprise }\end{array}$} & 1) Développer l'ergonomie du produit ou service \\
\hline & & $\begin{array}{l}\text { 2) Fournir des livrables concrets : plans, spécifications, maquettes, } \\
\text { résultats de tests, prototypes }\end{array}$ \\
\hline & & 3) Inclure un ou plusieurs pré-tests marketing \\
\hline & & $\begin{array}{l}\text { 4) Interroger des clients sur les fonctionnalités innovantes (focus group } \\
\text { ou panel) }\end{array}$ \\
\hline & & 5) Piloter par jalons \\
\hline & & $\begin{array}{l}\text { 6) Positionner le produit ou service développé par rapport à ceux } \\
\text { offerts par l'entreprise }\end{array}$ \\
\hline & & $\begin{array}{l}\text { 7) Positionner le produit ou service développé par rapport à ceux } \\
\text { utilisés par le client externe }\end{array}$ \\
\hline
\end{tabular}

\begin{tabular}{|l|l|l|}
\hline Stratégie & Famille associée & MC associé \\
\hline Résultat & $\begin{array}{l}\text { 12) Piloter le chiffre } \\
\text { d'affaires prévisionnel } \\
\text { du produit ou service } \\
\text { développé }\end{array}$ & $\begin{array}{l}\text { 1) Evaluer le chiffre d'affaires prévisionnel du produit ou service } \\
\text { développé à l'aide d'une étude de marché par exemple }\end{array}$ \\
\cline { 3 - 3 } & 2) Inclure un ou plusieurs pré-tests marketing \\
\hline
\end{tabular}

\begin{tabular}{|c|c|c|}
\hline Stratégie & Famille associée & MC associé \\
\hline \multirow[t]{3}{*}{ Résultat } & \multirow[t]{3}{*}{$\begin{array}{l}\text { 13) Piloter les coûts de } \\
\text { l'objet développé }\end{array}$} & $\begin{array}{l}\text { 1) Adapter la finesse de l'estimation des coûts à la durée du travail } \\
\text { nécessaire pour réaliser cette estimation }\end{array}$ \\
\hline & & 2) Collaborer directement avec les fournisseurs externes \\
\hline & & $\begin{array}{l}\text { 3) Intégrer les bases de données techniques et financières pour } \\
\text { automatiser la conception d'un produit ou service à coût cible }\end{array}$ \\
\hline
\end{tabular}




\begin{tabular}{|l|l|l|}
\hline \multirow{2}{*}{} & $\begin{array}{l}\text { 4) Recourir à une approche de gestion du coût du cycle de vie de } \\
\text { produit ou service s'appuyant sur le target costing }\end{array}$ \\
& $\begin{array}{l}\text { 5) Utiliser la comptabilité par activités pour faciliter la gestion } \\
\text { transverse des coûts prévisionnels }\end{array}$ \\
\hline
\end{tabular}




\section{Annexe 2: le dénombrement des Moyens de Contrôle observés longitudinalement sur ces quatre projets}

\begin{tabular}{|l|l|l|l|l|l|}
\hline Familles de Moyens de Contrôle & A & B & C & D & Total \\
\hline $\begin{array}{l}\text { 1) Favoriser la culture innovatrice des acteurs de } \\
\text { l'entreprise }\end{array}$ & 10 & 11 & 3 & 5 & 29 \\
\hline $\begin{array}{l}\text { 2) Contenir la différenciation des fonctions de } \\
\text { l'entreprise }\end{array}$ & 34 & 22 & 18 & 21 & 95 \\
\hline $\begin{array}{l}\text { 3) Rechercher l'intégration des fonctions de } \\
\text { l'entreprise }\end{array}$ & 64 & 39 & 36 & 25 & 164 \\
\hline 4) Gérer le slack & 9 & 11 & 5 & 11 & 36 \\
\hline $\begin{array}{l}\text { 5) Piloter la qualité au sein de l'entreprise en } \\
\text { concevant de façon pragmatique }\end{array}$ & 49 & 17 & 23 & 13 & 102 \\
\hline Sous-total processus & 108 & 72 & 57 & 51 & $\mathbf{2 8 8}$ \\
\hline 6) Evaluer le projet & 7 & 5 & 1 & 3 & 16 \\
\hline 7) Piloter l'allocation des ressources aux tâches & 20 & 11 & 9 & 5 & 45 \\
\hline 8) Piloter les coûts de projet & 8 & 9 & 9 & 1 & 27 \\
\hline $\begin{array}{l}\text { 9) Arbitrer entre des dimensions } \\
\text { Coûts/Délais/Qualités }\end{array}$ & 12 & 4 & 5 & 2 & 23 \\
\hline Sous-total arbitrage & 105 & 57 & 52 & 35 & $\mathbf{2 4 9}$ \\
\hline 10) Piloter les délais du projet & 7 & 6 & 13 & 3 & 29 \\
\hline $\begin{array}{l}\text { 11) Piloter la qualité perçue du produit ou service par } \\
\text { un client externe à l'entreprise }\end{array}$ & 54 & 17 & 24 & 18 & 113 \\
\hline $\begin{array}{l}\text { 12) Piloter le chiffre d'affaires prévisionnel du produit } \\
\text { ou service développé }\end{array}$ & 6 & 3 & 3 & 8 & 20 \\
\hline 13) Piloter les coûts de l'objet développé & 13 & 2 & 4 & 7 & 26 \\
\hline Sous-total résultat & $\mathbf{2 9 3}$ & $\mathbf{1 5 7}$ & $\mathbf{1 5 3}$ & $\mathbf{1 2 2}$ & $\mathbf{7 2 5}$ \\
\hline Total & & & & & \\
\hline
\end{tabular}

Tableau 12 : nombre de MC observés par famille de Moyens de Contrôle et par cas 